\title{
Consumer Profiling with Data Requirements: Structure and Policy Implications
}

\author{
Tommaso Valletti, Jiahua Wu \\ Imperial College Business School, Imperial College London, London, UK SW7 2AZ \\ \{t.valletti@imperial.ac.uk, j.wu@imperial.ac.uk\}
}

\begin{abstract}
We consider a model where a monopolist can profile consumers in order to price discriminate among them, and consumers can take costly actions to protect their identities and make the profiling technology less effective. A novel aspect of the model consists in the profiling technology: the signal that the monopolist gets about a consumer's willingness-to-pay can be made more accurate either by having more consumers revealing their identities, or by spending larger amounts of money (e.g., on third-party complementary data or data analytics capabilities). We show that both consumer surplus and social welfare are convex in the ability of consumers to conceal their identities. The interest of this result stems from the fact that consumers' concealing cost can be interpreted as a policy tool: a stricter privacy law would make the concealing cost lower, and vice-versa. Consequently, a policymaker who promotes total welfare should either make data protection very easy or very costly. The right direction of data regulations depends on data requirements. In particular, a higher (lower) data requirement is an instance when more (less) consumers are needed to achieve the same signal precision. We show that a strict data privacy law is preferable under a high data requirement so that firms are less likely to invest in profiling inefficiently, whereas there is less concern with little or no data regulations under a low data requirement. We also discuss when greater data protection may be beneficial to the firm.
\end{abstract}

Key words: profiling, privacy, price discrimination, signal accuracy, big data

\section{Introduction}

The explosion of information technologies has provided unprecedented ways for firms to collect data about their consumers. For instance, communications between web browsers and web sites allow firms to gather information including IP address, the web browser type, the computer model, as well as its operating system - all of which can be used in consumer profiling. Web sites can also assign and read unique identifiers, called cookies, which are used to compile records of individuals' browsing histories. Retail web sites like Amazon use cookies to keep track of what a consumer has shopped for and bought, and tailor web sites with products that the firm suspects that the 
consumer is the most interested in. Dansk Supermarked, Denmark's largest supermarket chain, partnered with Infosys - a leading IT consulting company - in order to use address data of its repeated consumers and tailor offers of products relevant to where they live. ${ }^{1}$ Uber introduced a new fare system called "route-based pricing", where fares are personalized based on its prediction of how much a customer is willing to pay. ${ }^{2}$ All these examples demonstrate that technological developments have enhanced a firm's ability to profile consumers and make them targeted offers.

On the other hand, consumers have become more wary with respect to how their information is being collected and used by firms. Singer (2015) reports several instances where consumers are aware of the many trade-offs associated with giving companies access to their data. Ultimately, the data can be supplied only by consumers themselves. The very same technologies that allow a firm to profile, can also be used by consumers to counteract the effectiveness of profiling. Consumers can remove their traces online by blocking third-party cookies, or purging cookie files. Some privacywary consumers even take further steps and pay third parties to protect their data. For example, Reputation.com charges individuals $\$ 9.95$ per month to remove personal data from on-line data markets. The search engine DuckDuckGo addresses searchers' privacy concerns by committing never to collect or share any individual information and search history.

Drawing upon this increasing tension between firms and consumers, we study data profiling in the context of price discrimination. While a large literature has analyzed consumer profiling and price targeting, which we review in Section 2, in this paper we consider the novel aspect of the accuracy of profiling. There are two entangled premises of our model, one related to actions that can be taken by the firm (data precision) and one related to actions that can be taken by consumers (data protection).

Suppose that a firm wants to profile its consumers by gathering and analyzing a dataset about them. The degree of precision of consumer's information is an endogenous choice of the firm, which can be improved in two complementary ways. On one hand, the precision of the information that can be extracted directly from consumers depends on the sample size. The relation between accuracy of statistical inference and sample size is well documented. For instance, in the classical linear regression, standard errors of Ordinary Least Squares estimators shrink at a rate of $\sqrt{n}$, where $n$ is the sample size. As a result, more accurate inferences could be drawn when the sample becomes larger. This assumption also echoes many practitioners' observation (e.g., Norvig 2011) that the

\footnotetext{
${ }^{1}$ https://www.infosys.com/industries/retail/case-studies/Documents/ecommerce-charge.pdf

${ }^{2}$ https://www.bloomberg.com/news/articles/2017-05-19/uber-s-future-may-rely-on-predicting-how-much-you-rewilling-to-pay
} 
scale of data needs to reach a minimal threshold to be sufficiently informative. Another way to improve precision is to invest either resources in acquiring data from third parties to complement the dataset about own consumers, or in data analysts and technologies to support them. ${ }^{3}$ A larger investment would result in an increase in the precision of consumers' information that one can potentially extract. For instance, firms in a wide range of industries, including grocery retailing, keep track of consumers' purchases over time via loyalty programs. On top of that, supermarkets not only collect information directly from their consumers, but also often supplement their consumer data by spending money on data collected from other sources, such as electoral rolls and credit reports. Tesco was the first UK supermarket to launch a loyalty scheme dated back to 1995. Later on, Tesco matched its own data with data from other sources, and created Crucible, a lucrative venture set up to allow other commercial organizations to pay for access to Tesco's data. ${ }^{4}$ With the help of loyalty programs, a firm is able to collect a panel data set of consumers, which allows the firm to control for time-invariant unobserved factors and facilitates causal inference in situations where inferring causality can be very difficult only with cross sectional data (Wooldridge 2010). Similarly, third-party data allows the firm to explicitly control for confounding factors in statistical analysis, again leading to more accurate inferences. While these two channels are both relevant and fit the purpose of improving the precision of the signal received about a consumer, they exhibit different features and therefore it is important to keep a distinction between them. Own data are likely to be idiosyncratic to the firm. Instead, data acquired from third parties (or investment in data analytics capabilities) are more akin to a general-purpose technology. This distinction plays an important role when it comes to the modelling, as shown later in the paper.

Turning to the second aspect of our model, consumers make endogenous choices whether to allow the firm to use information about them. A consumer can take a costly action to conceal his/her identity. This opens various interesting aspects that we contemplate. First, related to the point about sample size and accuracy, the firm needs to make sure that consumers endogenously prefer not to conceal their identities - otherwise the profiling techniques will be ineffective. This can be done either by offering them a good price in case they allow data disclosure, or by penalizing them in case they do not. Second, as information concealing is costly, there are welfare and policy questions arising. Regulators could make the cost of concealing smaller, for instance by imposing a full disclosure policy on the use of cookies. Conversely, this cost could be made larger if the firms

\footnotetext{
${ }^{3}$ Precision can also be obtained by buying another company that holds meaningful data, think of Facebook's acquisition of Instagram and WhatsApp that are planned to be integrated into a single platform. https://www.nytimes.com/2019/01/25/technology/facebook-instagram-whatsapp-messenger.html

${ }^{4}$ http://www.theguardian.com/business/2005/sep/20/freedomofinformation.supermarkets
} 
are allowed to trade consumers' data, so that consumers would need to request potentially many websites to erase their data. Hence we can also address, in a meaningful way, the question that is at the core of current debate on consumer privacy. How easy should access to consumers' information be?

Taking into account the factors described above, in this paper we consider a model where a monopolist seeks to price discriminate consumers through data profiling. Consumers can take costly actions to protect and anonymize their identities. Otherwise, a signal about a consumer's willingness-to-pay is received by the firm. The signal can be made more accurate either by having more consumers revealing their identities, or by investing larger amounts of money (e.g., on thirdparty data or data analytics capabilities).

We show that both consumer surplus and social welfare initially decrease as it becomes more difficult for consumers to anonymize their identities, however the relation is reversed when the cost of concealing becomes sufficiently large. The rationale is as follows. When it is very easy for consumers to conceal, it is sensible that consumer surplus would be relatively high because consumers can prevent the firm's profiling easily with a small cost. As concealing becomes more costly, more consumers would choose to reveal their identities, allowing the firm to profile them perfectly. However at the same time, the firm would charge a lower price to anonymous consumers, as this market becomes smaller with a greater proportion of low valuation consumers. So when the concealing cost becomes sufficiently large, the gain in surplus for anonymous consumers dominates the loss of surplus from those who reveal their identities, leading to an increase in consumer surplus in the concealing cost. Following a similar logic, social welfare also initially decreases, reaches its minimum, and then increases in the ability of consumers to conceal. In practice, consumers' concealing cost can be interpreted as a policy tool: a stricter privacy law would make the concealing cost lower, and vice-versa. Consequently, our result suggests that a policymaker who promotes total welfare should either make data protection very easy or very costly. The result that consumers may benefit from a very strict privacy law provides theoretical justification for the recent adoption of strict privacy regulations in many countries/regions. For instance, the General Data Protection Regulation ("GDPR") is a recent regulation in EU law on data protection and privacy, which significantly strengthened rights of individuals by making sure that individuals need to give explicit consent whenever businesses collect their data, and withdrawal of consent is as easy as giving consent. ${ }^{5}$ Somewhat more surprisingly, our result also suggests that total welfare can also be high at the other end of the spectrum when policymakers take little or no action over data privacy, ${ }^{5}$ https://eugdpr.org/ 
which is the stance some countries are taking right now. Having said that, privacy laws have significant impact on how consumer surplus is allocated across consumer segments. A strict privacy law mainly benefits those repeated customers who may participate in the firm's loyalty program, whereas new/switching customers are the main beneficiaries from the lack of privacy regulations.

Whether the firm's investment in profiling is aligned with social welfare is closely related to the ability of consumers to conceal their identities as well as to data requirements. In particular, we define a higher (lower) data requirement as an instance when more (less) consumers are required to achieve the same signal precision, for a given investment from the monopolist. When policymakers introduce very strict privacy laws such that consumers can easily conceal their identities, any of the firm's investment is rendered unprofitable, and the firm's decision not to profile is socially optimal, regardless of data requirement. Arguably, this is an extreme case in the sense that, by making it ineffective, it is as if profiling is being banned completely. In many cases, however, it is not realistic that a policymaker can affect the entire range of values of privacy cost. Often only piecemeal policy changes are implementable, and thus the policy maker could only affect privacy costs incrementally. Then, the optimal policy crucially hinges on data requirement of the business/application. For applications with relatively high data requirements, the policymaker shall introduce a relatively strict privacy law, which allows consumers to conceal easily. Coupled with the fact of high data requirements, it would more likely dissuade firms from inefficient investment in profiling, leading to socially optimal investment decisions from the firm. On the other hand, firms are already inclined to invest in profiling when data requirement is relatively low. In this case, policymakers should take little or no action over data privacy, such that consumers become less likely to spend inefficient effort trying to remove their information.

We further consider two extensions of our base model. First, we consider the case where consumers' concealing costs are heterogeneous. We show that, somewhat surprisingly, the firm's profit may decrease when a greater proportion of consumers have a high concealing cost. This is because the anonymous market becomes smaller with a greater concentration of low valuation consumers as the proportion of consumers with a high concealing cost increases, leading to a lower price for the anonymous market. As a result, the firm's profit from the anonymous market is maximized with a moderate proportion of consumers with a high concealing cost. So, even though the firm's profit from profiling always increases when more consumers find it costly to conceal their information, the reduction in profit from the anonymous market may outweigh the benefit from profiling, leading to a lower profit for the firm. In many cases, the firm itself is able to directly control the 
cost of maintaining anonymity. For instance, online retailers can determine how easy it is for consumers to delete cookies planted on their computers, or offer to delete consumer data periodically, as suggested in GDPR. Our result indicates that such practices may sometimes benefit the firm.

As a second extension, we consider the case when the firm's investment level is unobservable to consumers. Compared to the base model where the firm's investment level is observable, we show that either the two cases yield the same expected profit for the firm, or equilibrium exists only if the firm's investment level is observable. This is because, by committing to a certain investment level, the firm forces consumers to play along and respond optimally to a committed investment level.

\section{Literature Review}

Our paper relates to two broad streams in the literature. First, our paper is linked to the literature on behavior-based price discrimination. In the seminal work by Fudenberg and Tirole (2000), they study the effects of behavior-based price competition in the framework of a two-period Hotelling model. Firms are able to profile a consumer's preference on the Hotelling line based upon his/her purchase decision in the first period. Past behavior is then used in the second period to design a discriminatory pricing scheme by the firm. The research on behavior-based pricing has been extended to various settings and applications (Villas-Boas 1999, Villas-Boas 2004, Pazgal and Soberman 2008, Chen and Zhang 2009, Shin and Sudhir 2010, Zhang 2011). ${ }^{6}$ Comprehensive literature reviews can be found in Fudenberg and Villas-Boas (2007) and Esteves (2009). Most of the literature on behavior-based price discrimination does not consider privacy issue explicitly. The common recurring assumption in the literature is that a consumer has no option to remain anonymous once $\mathrm{s} /$ he purchased from the firm. In the context of our framework, it is equivalent to the case when consumers' concealing cost is extremely high, i.e., $c=1 / 2$, and thus it becomes prohibitive for a consumer to conceal his/her identity. Our work also differs from this stream of literature in that the granularity of profiling is an endogenous decision by the firm, whereas consumers can only be differentiated based on their purchasing history in the literature on behavior-based price discrimination.

The second stream of literature we are related to examines the implications of consumer privacy on pricing and privacy regulation, as well as their consequences on welfare (see Acquisti et al. 2016 for a comprehensive review of this literature). The majority of this stream of literature assumes that consumers' privacy decisions are exogenously determined (see, e.g., Taylor 2004, Acquisti and

\footnotetext{
${ }^{6}$ The literature on consumer addressability is also closely related (Chen et al. 2001, Chen and Iyer 2002).
} 
Varian 2005, Taylor and Wagman 2014, Shy and Stenbacka 2016). That is, discussions are typically centred around two special cases when it comes to privacy, namely, consumers either have no option to remain anonymous, which is the same as that in the literature on behavior-based price discrimination, or they can conceal/erase their data costlessly. In the context of our framework, the latter is equivalent to the case when concealing cost is extremely small, i.e., $c=0$. We contribute to the literature by exploring the entire spectrum of consumers' privacy cost, and show that, somewhat surprisingly, both consumer surplus and social welfare are convex in the privacy cost, implying that either a very strict privacy law or lack thereof can be good for consumers. We further explore the case when privacy costs are heterogeneous, and reveal that, surprisingly, the firm's profit may decrease when a greater proportion of consumers find it very costly to conceal their identities.

More recently, a growing number of works have considered the implications of consumers' endogenous decisions regarding how much information to be revealed to the firm. Casadesus-Masanell and Hervas-Drane (2015) consider a duopoly setting where consumers can choose the amount of information being provided to the firms. This information can help the firms to improve the quality of their products. Firms derive revenues from both consumer purchases and disclosure of consumer information in a secondary market. Montes et al. (2019) study the effects of price discrimination with endogenous consumers' privacy choices in the context of a duopoly Hotelling model. There is a data broker who collects consumers' information and can sell data to the two competing sellers. They show that the optimal selling strategy for the owner of consumer data is to deal with one firm exclusively. In the specific context of the auto insurance market, Chen and Jiang (2019) study the impact of in-vehicle monitoring technology on the insurance firms and drivers. The work closest related to ours is Conitzer et al. (2012), who study a monopolist's pricing problem in the framework of a two-period model. The monopolist is able to price discriminate consumers in the second period, depending on consumers' decisions regarding whether or not to purchase and whether or not to conceal their identities in the first period. Motivated by recent advance in data analytics, our work adds an important aspect to this discussion by allowing the firm to offer personalized prices to those identifiable consumers. This aspect considerably changes the impact of privacy cost on consumer surplus, as well as social welfare. On one hand, we obtain a result similar to Conitzer et al. (2012) that consumers may sometimes benefit from a high privacy cost. This is because, as privacy cost increases, the size of the anonymous market would become smaller with a greater proportion of low-valuation consumers. Consequently, the firm charges the anonymous market with a lower price, leading to higher consumer surplus as a result. However, unlike Conitzer et al. (2012), we show that consumer surplus may also decrease in the concealing cost, especially when the cost 
is very small. This decrease in consumer surplus is mainly driven by personalized prices offered by the firm, as the benefit from the ability of concealing with a lower cost becomes much larger.

To the best of our knowledge, two papers consider explicitly profiling technology in the context of price discrimination. In Koh et al. (2017), consumers can choose to disclose their private information to a monopolist in return for reduced search cost due to more accurate product recommendation. They face a trade-off between better product-fit and potential price discrimination. Belleflamme and Vergote (2016) study the optimal pricing of a monopolist who is able to profile consumers, while consumers are able to counteract by maintaining anonymity. What these two models have in common is that the signal received by the firm is assumed to follow a Bernoulli distribution when a consumer's true valuation is revealed with probability $\beta$, and no new information with probability $(1-\beta)$. Unlike the exogenously given profiling technology in Koh et al. (2017) and Belleflamme and Vergote (2016), our paper accounts for the firm's endogenous and costly investment in the precision of the signal. Coupled with the fact that consumers choose endogenously whether or not to reveal information, our framework adds an important dimension related to privacy regulation by accounting for the dynamics between data requirements and privacy cost. We show that the right privacy regulation hinges crucially on data requirements: total welfare typically benefits from a strict privacy law when data requirements are high, whereas lack of privacy law can be good for applications with low data requirements.

\section{The Model}

\subsection{The Setup}

A monopolist sells a product to a continuum of consumers with a total mass of one. There are two market segments, namely an "old market" and a "new market". The difference is that, in terms of consumer valuations, the firm only knows the aggregate distribution of the new market, while information can be obtained about individual consumers in the old market. We assume that the size of the old market is $\lambda$ and the size of the new market is $1-\lambda$, known to both the firm and consumers. In the following analysis, we use the subscripts $o$ and $n$ to denote the old market and the new market, respectively. We use the "old" vs "new" markets as a simple but flexible device to model different segments of consumers. The existence of the new market is crucial in our analysis. As we will show below, there exists a cutoff $\tilde{v}$ such that any consumer in the old market with a valuation greater than $\tilde{v}$ would choose to conceal and make herself anonymous. Knowing this, the firm would charge a price no lower than $\tilde{v}$ for anonymous consumers, without the new market. As a result, all consumers would be better off simply revealing their identities and receiving a non-negative utility. A separating equilibrium, where some consumers conceal, only exists when 
there is a market of new consumers. This assumption of the new market is also an appropriate reflection of reality, which we will discuss in details later in this section.

A consumer's valuation is determined by the realization of a random variable, which we assume to be uniformly distributed over the unit interval in both the new and the old market. A consumer in the old market can choose to perfectly conceal her identity to prevent the firm from profiling her valuation towards the product. A cost of $c(>0)$ is incurred if a consumer spends the effort to conceal her identity. We assume that, if a consumer is indifferent between revealing or concealing, she would choose to reveal her identity. ${ }^{7}$ Consumers in the new market and those consumers in the old market who conceal their identities constitute an anonymous market. That is, we assume that the monopolist cannot distinguish between new consumers (for whom no information is available) and old consumers who remove all available traces of their activities by paying the privacy cost. As a result, the firm can only offer one common uniform price to the anonymous market.

To put things into context, we can think of the firm as an online retailer. The new market can represent those consumers who are buying from the retailer's competitors, and thus have never shopped at the firm before. As such the retailer knows nothing about this segment of consumers. On the other hand, consumers in the old market can be repeated shoppers, who may also have joined the retailer's loyalty rewards program. For this segment, the firm is able to keep track of any individual's purchase history, her demographic information, etc., which can be used for profiling by the retailer. However, a repeated shopper can spend time and effort, for instance, registering a new account to remove her traces online. By doing so, the retailer will not be able to differentiate her from those consumers who may recently switch from competitors.

The firm invests a total amount of $K$ in collecting old consumers' information and profiling consumers. The investment in consumer profiling allows the firm to receive a signal, denoted as $s$, for each consumer in the old market who reveals her identity. The signal represents a mapping from the information about a consumer collected by the firm to a noisy prediction of the consumer' willingness to pay. The accuracy of the signal is assumed to be dependent on the firm's investment $K$, as well as the fraction of consumers choosing to reveal their identities, $\gamma$. That is, the degree of precision of the firm's prediction of consumers' willingness to pay can be improved in two complementary ways. On one hand, the firm is able to get more accurate signals when a greater proportion

\footnotetext{
${ }^{7}$ Alternatively, we can assume that a consumer always chooses to conceal her identity when she is indifferent between revealing or concealing. This would not change our results qualitatively, however the firm's and consumers' decisions at boundary cases will differ slightly. For instance, in Lemma 1 , the firm needs to invest more than $K_{o}$ to perfectly profile those consumers who choose to reveal, as a type- $v_{o}$ consumer in the old market would choose to conceal rather than reveal her identity. The rest remains the same.
} 
of consumers in the old market choose to reveal their valuations, which constitute the dataset that can be analyzed with statistical techniques. The justification of this assumption can be traced to the root of statistical inferences. A larger sample reduces the variation of sampling distribution, and thus predictions are more likely to be close to true values. With this assumption, we implicitly assume that there exists an externality in consumer profiling, because a consumer's decision of revealing her identity has implications for the rest of consumers in the dataset. This assumption also echoes many practitioners' observation (e.g., Norvig 2011) that the scale of data needs to reach a minimal threshold to be sufficiently informative. Typically, the relationship between the accuracy of signals and the amount of data available is S-shaped. On the other hand, more accurate signals are received with a higher investment $K$. The firm can invest in either obtaining additional data from third parties to supplement their consumer data, or more advanced data analytics for more accurate inferences. Note that these modeling features allow us to describe the process whereby digital platforms collect large amount of browsing data and process it through machine learning techniques. Perhaps the limit point of the long-term trajectory of this technological progress is best described by Larry Page, one of the founders of Google, in 2000: "Artificial intelligence would be the ultimate version of Google. The ultimate search engine that would understand everything on the web. It would understand exactly what you wanted, and it would give you the right thing. We're nowhere near doing that now. However, we can get incrementally closer to that, and that is basically what we work on." ${ }^{8}$ Instead, no profiling can be conducted in the new market, as no individual information is known to the firm.

The game unfolds in several stages. First, each consumer realizes her valuation $v$. The firm does not observe $v$; however, the uniform distribution of customers' valuations is common knowledge, known to both the firm and consumers. Second, the firm decides an investment level $K$ in consumer profiling. In the third stage, each consumer in the old market decides whether or not to conceal her identity. A cost of $c$ is incurred when concealing her identity. Next, the firm sets a base price to consumers in the new market, as well as to those in the old market who conceal their identities. The firm also offers a tailored price to each consumer in the old market who chooses to reveal her identity, based on the firm's belief of the consumer's valuation. Finally, each consumer makes the purchase decision, and she will purchase the product if and only if her utility from purchasing the product is non-negative.

${ }^{8}$ http://www.artificialbrains.com/google 


\subsection{Operationalization of Signal Accuracy}

Next we formalize the definition of accuracy of signals, which is operationalised by the conditional distribution of signals. Let $G(s \mid v)$ be the conditional cumulative distribution of signals from a type- $v$ consumer in the old market who chooses to reveal her identity. Its corresponding density function is denoted as $g(s \mid v)$. No signals would be received from those consumers who conceal in the old market, or in the new market. We specify the conditional density of signals from a type- $v$ consumer who reveals her identity as follows. For any $\alpha_{K, \gamma}>0$,

$$
g(s \mid v)= \begin{cases}\frac{1}{2 \alpha_{K, \gamma}} & \text { if } v-\alpha_{K, \gamma} \leq s \leq v+\alpha_{K, \gamma}, \\ 0 & \text { if } s<v-\alpha_{K, \gamma} \text { or } s>v+\alpha_{K, \gamma} .\end{cases}
$$

That is, the conditional distribution of signals still follows a uniform distribution. The mean of random signals equals to the consumer's valuation $v$, which is independent of the firm's investment as well as others' decisions. However, the conditional distribution rotates around the mean $v$ as $K$ and $\gamma$ varies. ${ }^{9}$ Consequently, the accuracy of the signal is determined solely by $\alpha_{K, \gamma}$. The signal $s$ degenerates to a constant of $v$, if $\alpha_{K, \gamma}=0$.

In our main analysis in Section $4, \alpha_{K, \gamma}$ is assumed to follow a step function. A more general specification of signals is considered in the complementary numerical analysis in Section 5.3, and insights do not change qualitatively. The specific functional form of $\alpha_{K, \gamma}$ is as follows:

$$
\alpha_{K, \gamma}= \begin{cases}+\infty & \text { if } K<\tau(\gamma) \\ 0 & \text { if } K \geq \tau(\gamma)\end{cases}
$$

That is, for a given fraction of consumers who choose to reveal their identities $\gamma$, if the investment from the firm is less than $\tau(\gamma)$, the firm is not able to gain any extra information by profiling those consumers. On the other hand, the firm is able to perfectly profile those consumers who reveal their identities, if the firm's investment reaches the threshold $\tau(\gamma)$.

The use of step functions is common in statistical analysis. One example is the classical hypothesis testing. In hypothesis testing, researchers first decide on a significance level, say $1 \%$ or $5 \%$, and then the test statistic is compared against the critical value corresponding to the chosen significance level. The null hypothesis is rejected if and only if the probability of observing more extreme values than the test statistic is less than the significance level, assuming that null is true. The firm's problem of consumer profiling can be considered as a variation of hypothesis testing problems, where the null hypothesis is that a consumer's valuation is equal to a pre-specified value. A more accurate estimate is obtained by having a larger sample. Or alternatively, the firm can invest to

\footnotetext{
${ }^{9}$ We note that the idea of rotation of distributions is similar to the one studied in Johnson and Myatt (2006).
} 
improve the statistical power of the test by controlling for more confounding variables or using more sophisticated methods. The assumption of $\alpha$ as a step function implies that the firm will act on the result of profiling if and only if the firm is sufficiently confident about the result.

We assume that $\tau(\gamma)$ is non-increasing and concave in $\gamma$. The non-increasing property of $\tau(\cdot)$ is consistent with the intuition that the firm's investment required to profile consumers perfectly decreases (weakly) in the fraction of consumers choosing to reveal their identities. The concavity of $\tau(\cdot)$ suggests that the required investment level decreases slowly when $\gamma$ is small, however the marginal effect of the size of dataset on the required investment increases when the dataset becomes larger. One way to understand the concavity of $\tau(\gamma)$ in $\gamma$ is as follows. The difference between $\tau\left(\gamma_{1}\right)$ and $\tau\left(\gamma_{2}\right)$, for any $\gamma_{1}>\gamma_{2}$, can be thought of as the firm's cost of buying an additional dataset which is equal to $\gamma_{1}-\gamma_{2}$ market share of the old market. For instance, $\tau(0.1)-\tau(0)$ is equal to the cost of buying data from $10 \%$ of the old market from third parties. When $\gamma$ is small, it is relatively cheap to purchase additional information from third parties. This is because the remaining market is large, and many consumers do not care much about their privacy, so their information may be widely available. As $\gamma$ increases, the pool becomes smaller, and the firm needs a more specific sample to complement its own dataset, which of course is more costly to gather. Thus the marginal impact of $\gamma$ on $\tau(\gamma)$ increases. We also assume that $\tau(1)>0$, i.e., under the situation when all consumers choose to reveal their identities, the firm still needs to commit a certain level of investment in order to profile the consumers perfectly. In the remainder, we will often work with the inverse function. If the firm's investment is $K$, the minimal fraction of consumers choosing to reveal their identities that allows the firm to perfectly profile consumers' valuations is given by $\tau^{-1}(K)$.

\section{Results}

In this section, we characterize the equilibrium, show comparative statics, and discuss implications of consumer profiling on consumer surplus and social welfare. We use Perfect Bayesian Equilibrium as the solution concept. Throughout the analysis, we impose the following assumption on the concealing cost to avoid the trivial equilibrium where concealing is so expensive that all consumers in the old market would choose to reveal their identities.

Assumption (C) (Concealing Cost) $c \leq 1 / 2$.

\subsection{Characterization of Equilibrium}

With $\alpha_{K, \gamma}$ being a step function, consumers' behavior in the old market can be characterized by the lemma below. 


\section{Lemma 1. (Consumers' Optimal Responses and Pricing) Denote}

$$
v_{o}=\frac{1}{2}+c+\frac{(1-2 c) \lambda}{2(2-\lambda)}, \quad \text { and } \quad K_{o}=\tau\left(v_{o}\right),
$$

where $v_{o}$ is increasing in both $\lambda$ and $c$, and $K_{o}$ is decreasing in both $\lambda$ and $c$.

(i) (Perfect Profiling) If $K \geq K_{o}$,

- a consumer in the old market chooses to conceal her identity if and only if $v>v_{o}$;

- the price the firm charges to the anonymous market is

$$
p^{*}\left(v_{o}\right)=\frac{1}{2}+\frac{\lambda\left(1-v_{o}\right)}{2(1-\lambda)}=\frac{1}{2}+\frac{(1-2 c) \lambda}{2(2-\lambda)} ;
$$

- the firm receives a perfect signal for any consumer in the old market who chooses to reveal her identity (i.e., $\alpha=0$ ), and charges an individual price equal to the signal.

(ii) (Non-Informative Profiling) If $\tau(1) \leq K<K_{o}$,

- a consumer in the old market chooses to conceal her identity if and only if $v \geq v_{K}=$ $\tau^{-1}(K)$

- the price the firm charges to the anonymous market is

$$
p^{*}\left(v_{K}\right)=\frac{1}{2}+\frac{\lambda\left[1-\tau^{-1}(K)\right]}{2(1-\lambda)} ;
$$

- signals from those who choose to reveal are non-informative (i.e., $\alpha=+\infty)$, and thus the firm offers one price to those who reveal their identities, which is given by $\tau^{-1}(K) / 2$.

(iii) If $K<\tau(1)$, all consumers in the old market would choose to conceal their identities. Signals are non-informative (i.e., $\alpha=+\infty$ ), and the firm would offer the optimal monopoly price $1 / 2$ to both the new market and the old market.

\section{Figure 1 Consumers' Optimal Responses under Various Investment Levels}

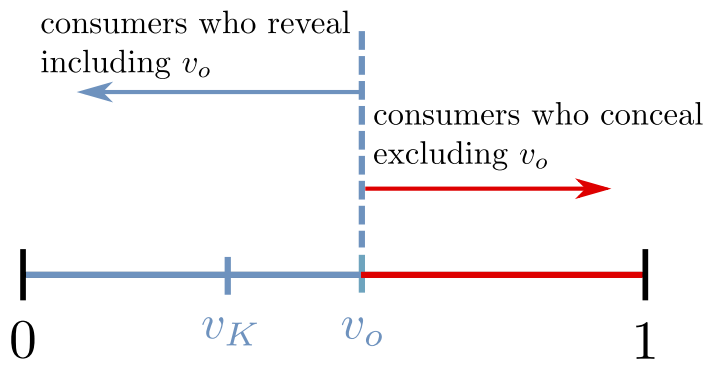

(a) $K \geq K_{o}$ (Perfect Profiling)

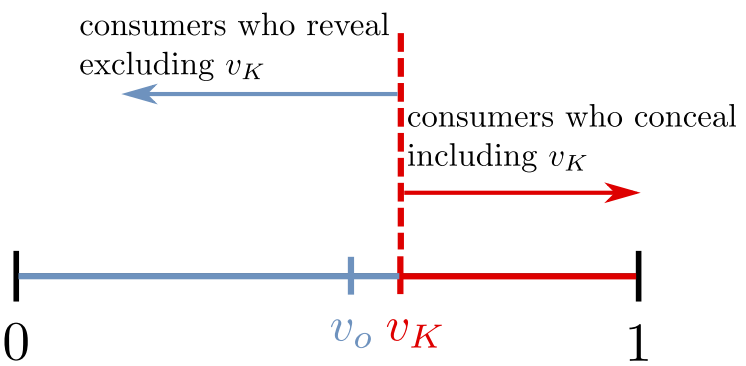

(b) $K \in\left[\tau(1), K_{o}\right)$ (Non-Informative Profiling)

Consumers' optimal response as a function of the firm's investment level is illustrated in Figure 1. In particular, the firm's investment levels can be divided into two regimes, depending on accuracy 
of signals from consumers who reveal in the old market. For any $K \geq K_{o}$, the firm receives perfect signals from consumers in the old market who choose to reveal (i.e., $\alpha=0$ ), and thus we refer to this scenario as "perfect profiling". On the contrary, for any $\tau(1) \leq K<K_{o}$, signals from those who choose to reveal are non-informative (i.e., $\alpha=+\infty$ ). This scenario will be referred to as "noninformative profiling". Lastly, there is a trivial case, when the firm's investment is less than $\tau(1)$. In this case, the firm cannot gain information even if all consumers in the old market choose to reveal their identities.

A few comments are in order. First, recall that a consumer from the old market needs to spend effort $c$ in order to conceal her identity. Intuitively, any consumer with a valuation lower than $c$ would find it unattractive to conceal her identity, and choose to simply reveal her identity. However, as we show in the proof of Lemma 1, at least a fraction $v_{o}$ of consumers in the old market would choose to reveal their identities. The logic is as follows. Anticipating a fraction $\tilde{v}$ of consumers in the old market revealing their identities, the firm would charge a price of $p^{*}(\tilde{v})=\frac{1}{2}+\frac{\lambda(1-\tilde{v})}{2(1-\lambda)}$ to the anonymous market, as shown in Lemma 1. As a result, the expected utility from concealing for any boundary consumer in the old market with valuation $\tilde{v}$ is given by $\max \left\{\tilde{v}-p^{*}(\tilde{v}), 0\right\}-c$, which increases in $\tilde{v}$ and is equal to zero when $\tilde{v}=v_{o}$. As the consumer's utility is non-negative simply revealing her identity, so the fraction of consumers who are guaranteed to reveal their identities is given by $v_{o}$, which is greater than $\frac{1}{2}+c$. Consequently, as long as the firm is able to commit an investment level of at least $K_{o}=\tau\left(v_{o}\right)$, it would gather sufficient data to profile consumers perfectly.

On the other hand, when the firm invests less than $K_{o}$, a consumer in the old market with valuation greater than $v_{o}$ may find it attractive to reveal her identity. In this case, she can save the cost from concealing her identity, and, at the same time, face a lower price from the firm, by mimicking decisions of consumers with lower valuations in the old market. However, as more consumers choose to reveal their identities, the firm is able to profile a larger proportion of the old market. As signal accuracy follows a step function, revealing a consumer's identity is strictly dominated by concealing when the proportion of consumers who choose to reveal is large enough to enable the perfect profiling from the firm. Thus, when $\tau(1) \leq K<K_{o}$, the amount of consumers who reveal will not be sufficient for the firm to receive perfect signals, as characterized in Lemma 1(ii).

Second, and perhaps surprisingly at first sight, no matter whether the firm is able to profile those who reveal perfectly, the price the firm charges to the anonymous market is always greater than $1 / 2$, which is the optimal monopoly price for the new market. The rationale is as follows. High 
valuation consumers from the old market would fear of being price discriminated as a result of the firm's profiling, and thus choose to conceal their identities. As a result, the valuation distribution of consumers in the anonymous market stochastically dominates the valuation distribution from the new market, leading to a higher price for the anonymous market.

Next we study the firm's optimal investment level in the first stage. Let $\pi_{K}(\lambda, c)$ denote the firm's profit with an investment level $K$. The lemma below summarizes the firm's profit at any given investment level.

LEMma 2. (FIRM's Profit) The firm's expected profit is given by

$$
\pi_{K}(\lambda, c)= \begin{cases}\frac{1}{2(2-\lambda)}+\frac{\lambda c^{2}(1-\lambda)}{2-\lambda}-K, & \text { if } K \geq K_{o}, \\ \frac{\lambda\left(v_{K}-1\right)^{2}}{4(1-\lambda)}+\frac{1}{4}-K, & \text { if } K \in\left[\tau(1), K_{o}\right), \\ \pi_{0}(\lambda, c)-K=\frac{1}{4}-K, & \text { if } K<\tau(1) .\end{cases}
$$

In particular, for any $K \in\left[\tau(1), K_{o}\right)$, the revenue contributed from the anonymous market is $\frac{\left(1-\lambda v_{K}\right)^{2}}{4(1-\lambda)}$, while the revenue from those who reveal their identities is $\frac{\lambda v_{K}^{2}}{4}$.

On one end of the spectrum, for any $K \geq K_{o}$, the fraction of consumers in the old market choosing to reveal their identities is given by $v_{o}$, which is independent of $K$, and the firm can profile those consumers perfectly. Thus, any investment beyond $K_{o}$ does not yield any extra revenue. On the other end of the spectrum, for any $K<\tau(1)$, the firm is not able to gain any information from profiling even if all consumers in the old market choose to reveal their identities. Thus, all consumers in the old market would be better-off revealing their identities, and the firm essentially faces two market segments, where the valuation distribution of consumers within each segment is uniformly distributed on the interval $[0,1]$. In this case, the firm's expected revenue is given by $1 / 4$.

The most interesting case is when $K \in\left[\tau(1), K_{o}\right)$. In this case, even though profiling becomes completely uninformative, the firm's investment would indirectly affect its profitability, as it shapes consumers' decisions as to whether to conceal their identities. As $K$ increases, the firm is able to force a larger proportion of consumers in the old market with high valuations to conceal, leading to a larger anonymous market with a greater proportion of high valuation consumers. At the same time, those consumers who choose to reveal their identities form a segment with relatively low valuations. This segment becomes smaller, with even lower valuations on average, as the firm's investment increases. Consequently, from the firm's perspective, consumers' endogenous concealing decisions as a response to the firm's investment in profiling creates an opportunity for third-degree 
price discrimination. As $K$ increases, the firm is able to better segment consumers in the old market, and thus extract higher revenue. This is reflected in Lemma 2 that the firm's revenue increases in $K$ for any $K \in\left[\tau(1), K_{o}\right)$. As discussed, a consequence of this third-degree price discrimination is that the new market would face a price higher than the optimal monopoly price, as suggested in Lemma 1.

Lemma 2 indicates that the firm's optimal investment level depends on curvature of $\tau(\cdot)$ function. However, as we show in the lemma below, it is reduced to two points, either 0 or $K_{o}$, under the assumption that $\tau(\cdot)$ is a concave function.

Lemma 3 (Optimal InVEstment LeVel). The firm's optimal investment level is either 0 or $K_{o}$.

Lemma 3 indicates that when the required level of investment is concave and decreasing in the fraction of consumers choosing to reveal their identities, the optimal solution is to either invest the minimum amount $K_{o}$ that allows the firm to profile consumers perfectly, or not to invest in consumer profiling at all. With an investment of $K_{o}$, the firm's optimal expected profit is given by $\pi_{K_{o}}(\lambda, c)=\frac{1}{2(2-\lambda)}+\frac{\lambda c^{2}(1-\lambda)}{2-\lambda}-K_{o}$. Due to $\lambda \in[0,1], \frac{1}{2(2-\lambda)} \geq \frac{1}{4}$ and $\frac{\lambda c^{2}(1-\lambda)}{2-\lambda}>0$, we obtain that the revenue from investing $K_{o}$ is always greater than $1 / 4$. As a result, the firm's decision on investing in consumer profiling depends ultimately on whether the increase in revenue outweighs the cost of profiling consumers. In a different context of corruptible markets, Singh (2017) shows a similar (in spirit) result that a buyer shall put forth either minimum or extensive effort to limit corruption when there exists a corruptible agent.

\subsection{Welfare Implications}

Whether or not the firm chooses to invest has different implications on consumer surplus (CS) and social welfare $(\mathrm{SW})$. With an investment of $K_{o}$, consumer surplus and social welfare are given by

$$
\begin{aligned}
C S_{K_{o}}(\lambda, c) & =\lambda \int_{v_{o}}^{1}\left[v-p^{*}\left(v_{o}\right)-c\right] d v+(1-\lambda) \int_{p^{*}\left(v_{o}\right)}^{1}\left[v-p^{*}\left(v_{o}\right)\right] d v \\
& =\frac{(2 \lambda-3)(1-\lambda c)^{2}}{2(2-\lambda)^{2}}+\frac{1+\lambda c^{2}}{2}-\lambda c \\
S W_{K_{o}}(\lambda, c) & =\lambda\left(\int_{0}^{v_{o}} v d v+\int_{v_{o}}^{1}(v-c) d v\right)+(1-\lambda) \int_{p^{*}\left(v_{o}\right)}^{1} v d v-K_{o} \\
& =\frac{1}{2}-\lambda c+\lambda c^{2}+\frac{\lambda c(1-\lambda c)}{2-\lambda}-\frac{(1-\lambda)(1-\lambda c)^{2}}{2(2-\lambda)^{2}}-K_{o} .
\end{aligned}
$$

Similarly, with a zero investment, consumer surplus and social welfare are given by

$$
C S_{0}(\lambda, c)=\lambda \int_{\frac{1}{2}}^{1}\left(v-\frac{1}{2}\right) d v+(1-\lambda) \int_{\frac{1}{2}}^{1}\left(v-\frac{1}{2}\right) d v=\frac{1}{8}
$$




$$
S W_{0}(\lambda, c)=\lambda \int_{\frac{1}{2}}^{1} v d v+(1-\lambda) \int_{\frac{1}{2}}^{1} v d v=\frac{3}{8} .
$$

Neither $\lambda$ nor $c$ plays a role in consumer surplus or social welfare when the firm invests zero. The reason is that with no investment from the firm, the signal from a consumer is non-informative. That is, the firm's posterior belief of a consumer's valuation is exactly the same as the prior belief. Consequently, any consumer in the old market would be better off revealing his identity, and the firm faces two identical markets in terms of the distribution of consumer valuations. However, if the firm invests, prices will differ and thus both $\lambda$ and $c$ affect the equilibrium. The impacts of $\lambda$ and $c$ on profit and consumer surplus are summarized in the lemma below.

Proposition 1. (Structural Properties)

(i) $\pi_{K_{o}}(\lambda, c)$ is increasing in both $\lambda$ and $c$;

(ii) $C S_{K_{o}}(\lambda, c)$ is decreasing in $\lambda$, and is convex in $c$;

(iii) $S W_{K_{o}}(\lambda, c)$ is convex in both $\lambda$ and $c$.

A few comments are in order. First, Proposition 1(i) indicates that the firm's profit increases in both $\lambda$ and $c$. This is intuitive in the sense that, when the size of the old market $\lambda$ increases, the firm is able to profile a larger proportion of the market, leading to a higher profit. Similarly, when consumers find it more difficult to conceal, a greater proportion of consumers in the old market would choose to reveal their identities, allowing the firm to extract greater surplus from them. A direct consequence of Proposition 1(i) is that the firm is more likely to invest in consumer profiling with either a higher $c$ or a higher $\lambda$.

Second, Proposition 1(ii) indicates that consumer surplus decreases in $\lambda$, as the firm can extract more surplus from consumers in the old market than the new market. Interestingly, consumer surplus is non-monotone in $c$. When $c$ is close to zero, consumers in the old market can conceal their identities easily, leading to relatively high consumer surplus. As $c$ increases, more consumers in the old market would choose to reveal their identities (as $v_{o}$ increases in $c$ ), allowing the firm to profile them perfectly. However at the same time, the price for the anonymous market decreases in $c$, i.e., $p^{*}\left(v_{o}\right)$ decreases in $c$, leading to an increase in consumer surplus from the anonymous market. Proposition 1(ii) suggests that the two forces combined lead to a convex structure of $C S_{K_{o}}(\lambda, c)$ in $c$. Following a similar logic, social welfare, which is equal to the sum of the firm's profit and consumer surplus, is also convex in $c$.

The interest in this discussion stems from the fact that $c$ could be interpreted as a policy tool: a stricter privacy law would make $c$ lower, and vice-versa. From this perspective, a policy 
maker that promotes total welfare should either make data protection very easy (low $c$ ) or very difficult (high $c$ ). The result that consumers may benefit from a very strict privacy law seems intuitive, and, as shown by Proposition 1, total welfare may also be relatively high as a result. This result provides theoretical justification for the recent adoption of strict privacy regulations in many countries/regions, such as GDPR in the EU, as discussed in the introduction. Somewhat more surprisingly, Proposition 1 suggests that total welfare can also be high at the other end of the spectrum when policymakers take little or no action over data privacy, which is the stance a few countries are taking right now. For instance, China has taken little action over data privacy compared to the west. Based on a survey released by the China Consumers Association, around $85 \%$ people had suffered some sort of data leak - ranging from their phone number being sold to spammers to their bank account details being stolen (Yang 2018). Even though total welfare can be relatively high with either very strict or no privacy laws, its implication on the allocation of consumer surplus across consumer segments differs significantly. From a consumer's perspective, a strict privacy law mainly benefits the old market (e.g., repeated customers who may participate in the firm's loyalty program), whereas the new market (e.g., new/switching customers) is the main beneficiary from the lack of privacy regulations. It follows that privacy laws can potentially be used as a policy tool to influence consumer behavior. For instance, the government of Japan is aiming to double Japan's cashless payment rates by 2025 due to reasons such as costly handling of cash, potential tax evasion with cash transactions, and etc. (Lewis 2019). However, Japan traditionally has a cash culture as people love the sense of security, ownership, as well as anonymity, that cash can bring to them. To facilitate its shift towards a cashless society, our result suggests that strict privacy enforcement with cashless payments is needed.

The impact of the firm's profiling investment on consumer surplus and social welfare is summarized in the corollary below.

Corollary 1. (Impact of Profiling on Consumer Surplus and Social Welfare) Comparing consumer surplus and social welfare under investment levels of 0 and $K_{o}$, we have

(i) for any $\lambda$ and $c, C S_{K_{o}}(\lambda, c) \leq C S_{0}(\lambda, c)$;

(ii) for any $\lambda>1 / 4$ and $c, S W_{K_{o}}(\lambda, c) \geq S W_{0}(\lambda, c)$ when $K_{o}$ is sufficiently small.

It is not surprising that investment in profiling enables the firm to capture more consumer surplus than it would otherwise without the investment. However, this investment is not necessarily socially optimal. If the firm chooses to invest in profiling consumers, the firm is able to sell to more consumers in the old market, especially to those with relatively low valuations due to personalized 
pricing. This is good for efficiency. At the same time, consumers with higher valuations would choose to spend effort to avoid price discrimination from the firm, leading to a loss in efficiency. Consequently, if the size of the old market is small, or the investment required to profile consumers perfectly, i.e., $K_{o}$, is high, investment leads to a suboptimal situation from the perspective of social welfare.

Having described how the equilibrium looks like, and having identified possible inefficiencies, we now ask a natural follow up and central question. What determines the extent to which the investment is socially optimal? Imagine a situation where prices to consumers are always set by the firm, but the investment level could be set by a social planner that maximizes total welfare instead of just the firm's profit. How does the investment level compare to that chosen by the firm? It turns out that whether the firm's investment is socially optimal depends critically on $\lambda$ and $c$. As shown in Lemma 4 below, if the required investment to perfectly profile consumers $K_{o}$ is greater than an upper threshold $\bar{K}_{\lambda, c}$, then it would be prohibitive for the firm to invest, and this decision turns out to be efficient. On the other end of the spectrum, if the amount of investment required is less than a lower threshold $\underline{K}_{\lambda, c}$, the firm prefers to invest in profiling consumers' valuations, and the increase in the sales outweighs the cost of investment $K_{o}$ and the amount of effort consumers spend to conceal their identities, leading again to a socially-optimal investment decision. However, for moderate $K_{o}$, the firm makes an excessive investment from the perspective of social welfare.

\section{Lemma 4. (Optimal Investment vs. Excessive Investment)}

(i) When $K_{o}>\bar{K}_{\lambda, c}$, the firm does not invest in consumer profiling, and this decision is socially optimal;

(ii) when $\underline{K}_{\lambda, c}<K_{o} \leq \bar{K}_{\lambda, c}$, the firm invests $K_{o}$ in consumer profiling, which leads to excessive investment from the perspective of social welfare;

(iii) when $K_{o} \leq \underline{K}_{\lambda, c}$, the firm invests $K_{o}$ in consumer profiling, and this decision is also socially optimal;

where $\bar{K}_{\lambda, c}=\frac{1}{2(2-\lambda)}+\frac{\lambda c^{2}(1-\lambda)}{2-\lambda}-\frac{1}{4}$ and $\underline{K}_{\lambda, c}=\frac{1}{8}-\lambda c+\lambda c^{2}+\frac{\lambda c(1-\lambda c)}{2-\lambda}-\frac{(1-\lambda)(1-\lambda c)^{2}}{2(2-\lambda)^{2}}$. In particular, the interval $\left(\bar{K}_{\lambda, c}-\underline{K}_{\lambda, c}\right)$ is: (1) increasing in $\lambda$; (2) concave in $c$, where its maximum and minimum are realized at $c=(1-\lambda) /(4-3 \lambda)$, and $c=1 / 2$, respectively.

As shown in Lemma 4, the interval where the firm's investment is inefficient becomes larger for a relatively larger old market. That is, when the size of the old market is large, the option of knowing consumers' valuations perfectly becomes more attractive for the firm because the firm is able to price discriminate a larger fraction of the market. Consequently, the chance of excessive investment becomes higher, especially when the investment required is high. 
Lemma 4 also shows that the interval $\left(\bar{K}_{\lambda, c}-\underline{K}_{\lambda, c}\right)$ is concave in $c$. It is easy to verify that $c=(1-\lambda) /(4-3 \lambda) \in[0,1 / 4]$ due to $\lambda \in[0,1]$. Consequently, starting from $c=1 / 2$, reducing the cost of concealing increases the width of the inefficiency interval leading to a higher chance of excessive investment from the perspective of total welfare. The width of the inefficiency interval is maximized at $c=(1-\lambda) /(4-3 \lambda)$, where any further reduction in the concealing cost from this point narrows the interval. Lemma 4 complements Proposition 1(iii) by showing that when it is very easy or very costly for consumers to conceal their identities, not only total welfare is likely to be high, the firm's decision to invest in profiling is also more likely to be socially optimal. Proof of Lemma 4 is available in Online Appendix.

\subsection{Impact of Data Requirements}

The final step in this section concerns the properties of the sampling technology that is used to profile consumers. We study how the firm's investment decision and profit will be affected by the different scenarios with respect to data requirement. There are situations when only "small data" are enough to generate statistically-relevant information about consumers, as opposed to truly "big data" instances whereby databases should include abundant information from many consumers. A way to think about a small-data case is one where there is a relatively simple and general statistical relationship in the population of consumers based on a few observables (think of gender and age, for instance). A few hundreds observations may suffice for the firm to unravel the relationship and understand its consumers with a very good degree of precision. Conversely, a situation about big data is one where there could be thousands of profiles, and the data requirements are orders of magnitude larger. ${ }^{10}$

The definition below sets the stage for our discussion. In particular, we say one scenario $\tau_{1}$ indicates higher data requirement than another scenario $\tau_{2}$, if $\tau_{1}(\gamma) \geq \tau_{2}(\gamma), \forall \gamma \in[0,1]$. That is, with the same investment from the firm, a larger fraction of consumers is required in order to profile their valuations perfectly under $\tau_{1}$ than that under $\tau_{2}$. An alternative way to interpret the definition is that, for the same fraction of consumers who reveal their identities, a higher investment is needed from the firm under $\tau_{1}$ to profile consumers perfectly.

Definition 1. (Higher Data Requirement) For two functions $\tau_{1}$ and $\tau_{2}, \tau_{1}$ represents a scenario with higher data requirement than $\tau_{2}$ if $\tau_{1}(\gamma) \geq \tau_{2}(\gamma), \forall \gamma \in[0,1]$ and the inequality is strict for some $\gamma$.

\footnotetext{
${ }^{10}$ According to the Federal Trade Commission, one data broker's database has information on 1.4 billion consumer transactions and over 700 billion aggregated data elements (Federal Trade Commission 2014). Although related to a different setting, a "big data" problem is also the Netflix prize. In 2009, Netflix awarded a $\$ 1 \mathrm{~m}$ prize for the best filtering algorithm to predict user ratings for films. A data set of 100,480,507 ratings that 480,189 users gave to 17,770 movies was provided by Netflix. See https://en.wikipedia.org/wiki/Netflix_Prize.
} 
The implications of higher data requirement on the firm's profit, consumer surplus and social welfare are summarized in the proposition below. Given the same fraction of consumers who choose to reveal their identities, the amount of investment required is lower with lower data requirement: hence the firm is more likely to invest in consumer profiling, and the firm's profit is always higher under a scenario with lower data requirement. On the other hand, because the firm's investment always leads to lower consumer surplus as shown in Corollary 1, consumer surplus is thus lower when the data requirement is lower.

Proposition 2. (Impact of Data Requirement) Consider two scenarios $\tau_{1}$ and $\tau_{2}$, where $\tau_{1}$ indicates higher data requirement. Then,

(i) the firm is more likely to invest in profiling under $\tau_{2}$, and the firm's optimal profit is also higher under $\tau_{2}$;

(ii) consumer surplus is (weakly) lower under $\tau_{2}$;

(iii) with small c, the firm's investment decision is socially optimal under both scenarios; with large $c$, the firm's investment decision is more likely to be efficient under $\tau_{2}$; with moderate $c$, the firm's investment decision is more likely to be efficient under $\tau_{1}$.

The impact of data requirement on social welfare is the most involved and deserves further comment. Recall that the firm's profit when investing in consumer profiling is always increasing in $c$. Consequently, with small $c$, the firm does not invest under either a high-data-requirement scenario or a low-data-requirement scenario, and the firm's decision is efficient under both scenarios. With large $c$, the firm invests under both scenarios, and its investment decision is more likely to be socially optimal under a scenario with lower data requirement due to the lower amount of investment required. With moderate $c$, the firm will invest when the data requirement is low, but does not invest otherwise. In this case, the decision of no investment under high data requirement is guaranteed to be efficient, while the decision of investment may be excessive if the condition shown in Lemma 4(ii) is satisfied.

Proposition 2(iii) has important policy implications. In the case when a policymaker introduces very strict privacy laws, any of the firm's investment is rendered unprofitable, and the firm's decision of not profiling in this case is socially optimal, regardless of data requirement. Arguably, this is an extreme case in the sense that it is as if profiling is being banned completely. Some policymakers are indeed adopting this view regarding privacy. An example is the recent ban on the use of facial recognition technology by police and other agencies in San Francisco (Conger et al. 2019). This ban essentially reduces $c$ to 0 , and thus all individuals can remain anonymous costlessly. Having 
said that, in many cases, it is not realistic that a policymaker can affect the entire range of values of $c$. Often only piecemeal policy changes are implementable, and thus the policy maker could only affect privacy costs incrementally. Proposition 2(iii) shows that the optimal policy crucially hinges on data requirements of the particular business/application. For applications with high data requirements, the firm's action is more likely aligned with that of the central planner under strict privacy laws. On the other hand, policymakers can get away with little or no privacy regulations for applications with a relatively low data requirement.

\section{Extensions}

\subsection{Heterogeneous Concealing Costs}

In the base model, we assume that all consumers in the old market incur an identical cost if they choose to conceal their identities. Arguably, this assumption is restrictive. For instance, consumers may differ in their capabilities of removing their traces online. Some tech-savvy consumers might find it easier to delete cookies implanted on their PCs or use proxy software to mask their IP addresses. This cohort of consumers effectively face a lower concealing cost than others. In this section, we extend our base model by accounting for consumers' heterogeneous concealing costs.

The setup of the model is the same as the base model, with the only exception that there are now two segments of consumers in the old market. In particular, $\theta_{H}$ proportion of customers in the old market has a high concealing cost of $c_{H}$, and $\theta_{L}$ proportion of customers has a low concealing cost of $c_{L}<c_{H}$. These two segments make up the old market, i.e., $\theta_{H}+\theta_{L}=1$. Similar to the base model, we assume the following on concealing costs to avoid any trivial solution where one segment of consumers would always prefer to reveal their identities than to conceal. As we will show in Lemma 5 , this assumption guarantees that $v_{o}^{H} \leq 1$.

Assumption (HC) (Heterogeneous Concealing Costs) $c_{L} \leq c_{H} \leq \frac{1-\left(1-c_{L} \theta_{L}\right) \lambda}{2-\left(1+\theta_{H}\right) \lambda} \cdot{ }^{11}$

We first study consumers' optimal responses and the firm's pricing strategy, given any investment level $K$. Results are summarized in the lemma below. Proofs of results in this section are available in Online Appendix.

Lemma 5. (Consumers' Optimal Responses and Pricing) Denote

$$
v_{o}^{i}=\frac{1}{2}+c_{i}+\frac{\lambda}{2(2-\lambda)}\left(1-2 c_{H} \theta_{H}-2 c_{L} \theta_{L}\right), i \in\{L, H\}, \quad \text { and } \quad K_{o}^{\prime}=\tau\left(\theta_{H} v_{o}^{H}+\theta_{L} v_{o}^{L}\right)
$$

(i) (Perfect Profiling) If $K \geq K_{o}^{\prime}$,

\footnotetext{
${ }^{11}$ When $c_{L}=c_{H}=c$, Assumption (HC) boils down to Assumption (C) $c \leq 1 / 2$ as specified in Section 4 .
} 
- for any consumer with concealing cost $c_{i}$, she would conceal her identity if and only if $v>v_{o}^{i}, i \in\{L, H\}$;

- the price the firm charges to the anonymous market is

$$
p^{*}\left(v_{o}^{H}, v_{o}^{L}\right)=\frac{1}{2}+\frac{\theta_{H} \lambda\left(1-v_{o}^{H}\right)+\theta_{L} \lambda\left(1-v_{o}^{L}\right)}{2(1-\lambda)}=\frac{1}{2}+\frac{\lambda}{2(2-\lambda)}\left(1-2 c_{H} \theta_{H}-2 c_{L} \theta_{L}\right) ;
$$

- the firm receives a perfect signal for any consumer in the old market who chooses to reveal her identity, and charges an individual price equal to the signal.

(ii) (Non-Informative Profiling(I)) If $\tau\left(1-\theta_{L}\left(c_{H}-c_{L}\right)\right) \leq K<K_{o}^{\prime}$,

- for any consumer with concealing cost $c_{i}, i \in\{L, H\}$, she would conceal her identity if and only if $v \geq v_{K}^{i}, i \in\{L, H\}$;

- the price the firm charges to the anonymous market is

$$
p^{*}\left(v_{K}^{H}, v_{K}^{L}\right)=\frac{1}{2}+\frac{\theta_{H} \lambda\left(1-v_{K}^{H}\right)+\theta_{L} \lambda\left(1-v_{K}^{L}\right)}{2(1-\lambda)}=\frac{1-\lambda \tau^{-1}(K)}{2(1-\lambda)} ;
$$

- signals from those who choose to reveal are non-informative, and thus the firm offers one price to those who choose to reveal, which is given by

$$
p_{s}^{*}=\frac{\theta_{H} v_{K}^{H}+\theta_{L} v_{L}^{H}}{2}=\frac{\tau^{-1}(K)}{2},
$$

where $v_{K}^{H}=\tau^{-1}(K)+\theta_{L}\left(c_{H}-c_{L}\right)$ and $v_{K}^{L}=\tau^{-1}(K)-\theta_{H}\left(c_{H}-c_{L}\right)$.

(iii) (Non-Informative Profiling(II)) If $\tau(1) \leq K<\tau\left(1-\theta_{L}\left(c_{H}-c_{L}\right)\right.$ ),

- all consumers with high concealing cost would reveal their identities, i.e., $v_{K}^{H}=1$;

- a consumer with low concealing cost would conceal her identity if and only if $v \geq v_{K}^{L}=$ $\frac{\tau^{-1}(K)-\theta_{H}}{\theta_{L}}$

- the price the firm charges to the anonymous market is $p^{*}\left(v_{K}^{H}, v_{K}^{L}\right)=\frac{1-\lambda \tau^{-1}(K)}{2(1-\lambda)}$;

- signals from those who choose to reveal are non-informative, and thus the firm offers one price to those who choose to reveal, which is given by $p_{s}^{*}=\frac{\tau^{-1}(K)}{2}$.

(iv) If $K<\tau(1)$, all consumers in the old market would choose to conceal their identities. Signals are non-informative, and the firm would offer the optimal monopoly price $1 / 2$ to both the new market and the old market.

Comparing Lemma 5 against consumers' optimal responses in the base model, as characterized in Lemma 1, we notice that consumers' optimal responses are similar in structure under the two cases. When the firm's investment is greater than $K_{o}^{\prime}$, a sufficiently large proportion of consumers in the old market would prefer to reveal their identities. As such, signals are perfect, and the firm 
can perfectly price discriminate those consumers who choose to reveal. Lemma 5(i) also shows that a greater proportion of consumers with a high concealing cost would prefer to reveal their identities compared to consumers with a low concealing cost, i.e., $v_{o}^{H} \geq v_{o}^{L}$. This is intuitive in the sense that a consumer is more likely to reveal her identity if it is more costly for her to conceal. As a matter of fact, the difference in the proportions of consumers who choose to reveal in the two segments is equal to the difference of concealing costs, i.e., $v_{o}^{H}-v_{o}^{L}=c_{H}-c_{L}$. The composition of the old market would affect the required investment level for perfect profiling $K_{o}^{\prime}$, as well as the firm's pricing policy. As the proportion of consumers with a high concealing cost $\theta_{H}$ increases, the firm would be able to achieve perfect profiling with a lower investment level, and charge a lower price to the anonymous market.

When $K \in\left[\tau(1), K_{o}^{\prime}\right)$, infinite number of equilibria could potentially exist, as an equilibrium only requires that $v_{K}^{H}>v_{o}^{H}$ and $v_{K}^{L}>v_{o}^{L}$. We focus on a symmetric equilibrium which requires $v_{K}^{H}=v_{K}^{L}$ when $c_{H}=c_{L}$, as this is a natural extension of the equilibrium from the base model. In other words, the equilibrium from this refinement is directly comparable to that of the base model. It is easy to verify that, as $K$ decreases, more consumers, in both segments with a high concealing cost and a low concealing cost, would prefer to reveal their identities. We further differentiate between two cases, namely $v_{K}^{H}<1$ and $v_{K}^{H}=1$. When $v_{K}^{H}<1$, which happens when the firm's investment level $K>\tau\left(1-\theta_{L}\left(c_{H}-c_{L}\right)\right)$, the difference of proportions of consumers who reveal in the two segments is equal to the difference of concealing costs, i.e., $v_{K}^{H}-v_{K}^{L}=c_{H}-c_{L}$. Only part of consumers choose to reveal in both segments in the old market, as characterized in Lemma 5(ii). Whereas when $v_{K}^{H}=1$, which happens when the firm's investment $K \leq \tau\left(1-\theta_{L}\left(c_{H}-c_{L}\right)\right)$, all consumers with a high concealing cost would reveal their identities. In both cases, because fewer consumers in both segments would choose to reveal their identities as the firm's investment $K$ increases, the firm would charge a higher price to the anonymous market, but a lower price to those who reveal their identities.

Lastly, there is also a trivial case when $K<\tau(1)$. This case is exactly the same as that of the base model, where all consumers in the old market would reveal their identities, and the firm offers the optimal monopoly price $1 / 2$ to both the new market and the old market.

Proposition 3. (FIrm's Profit) With heterogeneous concealing costs, the firm's expected profit is given by 


$$
\pi_{K}(\lambda, c)= \begin{cases}\frac{1+\lambda(2-\lambda)\left(c_{L}^{2} \theta_{L}+c_{H}^{2} \theta_{H}\right)-\lambda^{2}\left(c_{L} \theta_{L}+c_{H} \theta_{H}\right)^{2}}{2(2-\lambda)}-K, & \text { if } K \geq K_{o}^{\prime}, \\ \frac{\lambda\left(\tau^{-1}(K)-1\right)^{2}}{4(1-\lambda)}+\frac{1}{4}-K, & \text { if } K \in\left[\tau(1), K_{o}^{\prime}\right), \\ \frac{1}{4}-K, & \text { if } K<\tau(1) .\end{cases}
$$

In particular, consider the scenario when $K \geq K_{o}^{\prime}$. If either $\lambda \leq 2 / 3$, or $\lambda>2 / 3$ and $c_{H} \leq \frac{2-\lambda}{3 \lambda-2} c_{L}$, $\pi_{K}(\lambda, c)$ increases in $\theta_{H}$ for any $0 \leq \theta_{H} \leq 1$. However, if $\lambda>2 / 3$ and $c_{H}>\frac{2-\lambda}{3 \lambda-2} c_{L}$, there exists $\theta_{H}^{*}=\frac{(2-\lambda) c_{H}+(2-3 \lambda) c_{L}}{2 \lambda\left(c_{H}-c_{L}\right)} \in(0,1)$ such that $\pi_{K}(\lambda, c)$ increases in $\theta_{H}$ for any $\theta_{H} \leq \theta_{H}^{*}$, but decreases in $\theta_{H}$ otherwise.

Proposition 3 summarizes the firm's optimal expected profit given any investment level $K$. When $K<K_{o}^{\prime}$, the firm's profit is similar to that of the base model when consumers' concealing cost is homogeneous. In this case, the firm's expected profit is independent of the composition of the old market, because the fraction of consumers in the old market who choose to reveal remains the same.

When $K \geq K_{o}^{\prime}$, interestingly, the firm's profit is not guaranteed to be increasing in $\theta_{H}$ at any given investment level $K$. This contradicts the result from the base model that the firm's profit always increases in $c$ for any $K \geq K_{o}$, as shown in Lemma 3. The rationale is as follows. As $\theta_{H}$ increases, the fraction of consumers in the old market who choose to reveal their identities, i.e., $\theta_{H} v_{o}^{H}+\theta_{L} v_{o}^{L}$, increases. Because the firm can profile those consumers perfectly for any $K \geq K_{o}^{\prime}$, the firm's profit from those who choose to reveal always increases in $\theta_{H}$. On the other hand, as $\theta_{H}$ increases, the size of the anonymous market becomes smaller, with a greater proportion of consumers of low valuations. Thus, the firm charges a lower price to the anonymous market as $\theta_{H}$ increases. Overall, the firm's expected revenue from the anonymous market is concave in $\theta_{H}$. Under the conditions that the old market is sufficiently large, i.e., $\lambda>2 / 3$, and the discrepancy in the concealing costs is not too small, i.e., $c_{H}>\frac{2-\lambda}{3 \lambda-2} c_{L}$, there is a region of $\theta_{H}$ in which the loss from the anonymous market outweighs the gain from the perfect profiling of those who reveal as $\theta_{H}$ increases.

In many cases, the firm itself is able to directly control the cost of maintaining anonymity. For instance, online retailers can determine how easy it is for consumers to delete cookies planted on their computers, or offer to delete consumer data periodically as suggested in GDPR. Proposition 3 shows that the firm may sometimes be better-off if it allows more customers to remain anonymous more easily. This is great news for firms, especially those operating under the conditions specified in the preceding paragraph. By allowing consumers to control their privacy more easily, the firm not 
only establishes a good corporate image by showing that it really cares about consumer privacy, but also earns a higher profit at the same time.

\subsection{Unobservable Investment from the Firm}

In the base model, the firm's investment is assumed to be observable to consumers, and, in turn, consumers decide whether or not to conceal their identities in response. This assumption is reasonable in many situations. For instance, as in the examples discussed in Section 1, firms' investments in data analytics are commonly reported by press and media, which are thus made available to consumers. These investment decisions are costly and cannot be adjusted easily. Having said that, as nowadays consumers become more wary with respect to how their information is being collected and used, firms do have an incentive to hide their profiling investment, with the hope that more consumers would reveal their identities. Thus, in this section, we consider a model where the firm's investment is unobservable to consumers.

In this case, it would be as if the firm and consumers make decisions simultaneously. For any level of firm's investment $K$, the best response from consumers is still characterized by Lemma 1 . On the other hand, we can characterize the optimal simultaneous choice of investment from the firm as follows. Proofs of results in this section are available in Online Appendix.

Lemma 6. (FIRM's BeSt REPONSE) Expecting a type-v customer in the old market would conceal if and only if $v>\tilde{v}$, the firm's best response is:

(i) If $\tilde{v}<v^{\prime}$ or $v^{\prime}$ does not exist, the firm is better off not investing at all, i.e., $K(\tilde{v})=0$;

(ii) If $\tilde{v} \geq v^{\prime}$, it is optimal for the firm to invest $K(\tilde{v})=\tau(\tilde{v})$,

where $v^{\prime}$ is the unique solution of $\tau(\tilde{v})=\frac{\lambda \tilde{v}^{2}}{4}$, if it exists.

Lemma 6 shows that, anticipating that $\tilde{v}$ fraction of consumers in the old market would reveal their identities, the firm's optimal investment level would be either 0 or $\tau(\tilde{v})$. For any $K<\tau(\tilde{v})$, signals from consumers are completely uninformative, and thus expected revenue remains the same for any $K$. Thus, the firm is better off investing 0 . On the other hand, any investment greater than $\tau(\tilde{v})$ cannot be optimal either, as the firm can already perfectly profile those who reveal with $K=\tau(\tilde{v})$. Thus, the firm would either invest $\tau(\tilde{v})$ or not invest at all. Intuitively, the former dominates the latter when a large fraction of consumers choose to reveal their identities. Lemma 6 shows that this holds if and only if $\tilde{v} \geq v^{\prime}$.

We can derive the equilibrium by studying the best response curves from consumers and the firm, as characterized in Lemma 1 and Lemma 6, respectively. The result is summarized below. 
Proposition 4. (Equilibrium under Unobservable Investment) When the firm's investment is unobservable, the equilibrium can be characterized as follows.

(i) When $v^{\prime} \leq v_{o}$, there exists a unique equilibrium where the firm invests $K_{o}$, and consumers' response and the firm's pricing policy are characterized in Lemma 1(i);

(ii) When $v^{\prime} \in\left(v_{o}, 1\right]$, there exists no equilibrium;

(iii) When $v^{\prime}>1$ or $v^{\prime}$ does not exist, there exists a unique equilibrium where the firm does not invest in profiling, i.e., $K=0$, and consumers' response and the firm's pricing policy are characterized in Lemma 1(iii).

In particular, $K_{o}$ and $v_{o}$ are given in Lemma 1 , and $v^{\prime}$ is given in Lemma 6 .

Comparing Proposition 4 against the equilibrium when the firm's investment is observable, which is characterized by Lemma 1 and Lemma 3, the two cases yield identical outcome when $v^{\prime} \leq v_{o}$, or when $v^{\prime}>1$ or $v^{\prime}$ does not exist. However, when $v^{\prime} \in\left(v_{o}, 1\right]$, an equilibrium exists only if the firm's investment level is observable, as by committing to a certain investment level, the firm forces consumers to play along and respond optimally to the given investment level.

\subsection{General $\alpha_{K, \gamma}$}

We managed to get several interesting insights analytically in Section 4. In this section, we adopt a more flexible logistic specification for $\alpha_{K, \gamma}$, and further supplement our analytical results with numerical analysis. In particular, $\alpha_{K, \gamma}$ is specified as follows:

$$
\alpha_{K, \gamma}=\frac{1}{1+\exp \left\{a\left(\gamma-b+K^{d}\right)\right\}},
$$

where $a \geq 0$ and $d \geq 0$. It is easy to verify that $\alpha_{K, \gamma}$ as given by Equation (1) is decreasing in $\gamma$ and $K$, which is consistent with our assumption that more accurate signals are received with a higher investment $K$ or a greater proportion of consumers $\gamma$ who choose to reveal their valuations. When $a \rightarrow \infty, \alpha_{K, \gamma}$ degenerates to a step function. Consequently, studying this more general $\alpha_{K, \gamma}$ allows us to verify our findings, as well as explore the regimes that would be infeasible in Section 4 .

The equilibrium analysis under the general $\alpha_{K, \gamma}$ can be found in Online Appendix. In summary, we can no longer characterize consumers' decisions on whether or not to conceal, and the firm's pricing decisions at equilibrium with closed-form analytical results. Thus, we resort to numerical analysis. We show below one set of results on the impact of data requirements. Additional results on how other forces would affect the firm's profit, consumer surplus and social welfare are available in Online Appendix.

Figure 2 illustrates the impact of $b$ and $d$ on the optimal investment level $K$, firm's optimal profit $\pi_{K}(\lambda, c)$, consumer surplus $C S_{K}(\lambda, c)$, and social welfare $S W_{K}(\lambda, c)$. In general, a higher $b$ 
Figure 2 Impact of $b$ and $d$ on Optimal Investment Level $K$, Firm's Optimal Profit $\pi_{K}(\lambda, c)$, Consumer Surplus $C S_{K}(\lambda, c)$, and Social Welfare $S W_{K}(\lambda, c)$.

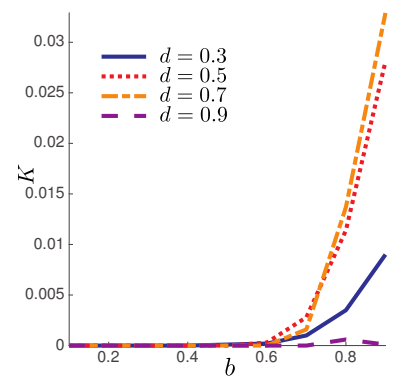

(a) $K$

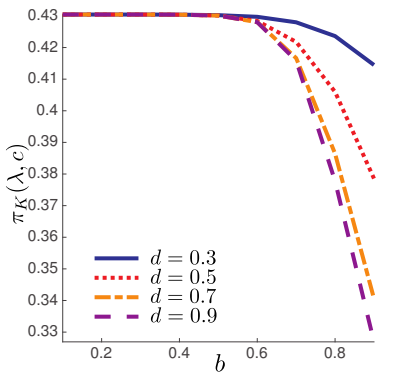

(b) $\pi_{K}(\lambda, c)$

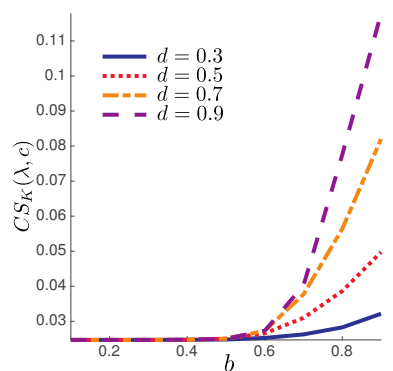

(c) $C S_{K}(\lambda, c)$

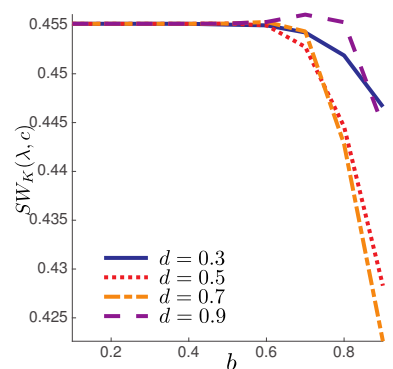

(d) $S W_{K}(\lambda, c)$

Note. The parameters are specified as follows. $c=0.25, \lambda=0.7$ and $a=20$.

(or a higher $d$ when $K<1$ ) indicates a scenario with higher data requirement, other things being equal (please see Online Appendix for a detailed discussion). Figure 2(a) suggests that the optimal investment $K$ is not monotone in the level of data requirement. When the data requirement is low, a small investment is sufficient for the firm to profile consumers with good accuracy, and thus the optimal investment from the firm tends to be small. As data requirement increases, the firm generally increases its investment level with the hope of more accurate signals from consumers. At the same time, the fraction of consumers who choose to reveal their identities decreases. Consequently, when the data requirement is sufficiently high, even a high investment would not allow the firm to profile consumers accurately due to the lack of the scale of data, leading the firm to scale back its optimal investment level.

Though $K$ is not monotone in either $b$ or $d$, Figure 2(b) suggests that the firm's profit $\pi_{K}(\lambda, c)$ is monotonically decreasing in both $b$ and $d$. That is, a higher data requirement is always detrimental to the firm's profitability, which is consistent with our result under the special case shown in Proposition 2(i). Similarly, consumer surplus $C S_{K}(\lambda, c)$ increases in both $b$ and $d$ as illustrated in Figure 2(c), which is a direct extension of Proposition 2(ii). Since profits decrease and consumer surplus increases, it is not surprising that total welfare is not monotone in the level of data requirement, as shown in Figure 2(d).

\section{Conclusion}

In this paper, we study data profiling in the context of price discrimination. Our main contribution to the literature is the novel focus on two endogenous and related decisions: the firm invests in the precision of the information it gets from consumers, while consumers can take costly actions 
to protect their privacy. We show that the optimal privacy policies and regulations closely relate to the flexibility of consumers to conceal their identities as well as to data requirements.

A policymaker who promotes total welfare should either make data protection very easy or very costly. Consumers from the old targeted market benefit from stricter data protections, because otherwise they are negatively affected from price discrimination. However, the anonymous market can benefit from little or no data protections, because the firm would charge them a lower price when it becomes more difficult for consumers in the old targeted market to conceal. The optimal policy crucially hinges on data requirement of the business/application. When it is easy for consumers to protect their data, private and social incentives are aligned when data analytics involve a large data requirement. On the other hand, when it is very costly for consumers to conceal their information, a small data requirement induces an investment on the firm's side that is very close to that would be chosen by a social planner.

We consider a monopoly model in this paper. This is a reasonable assumption in many situations with dominant platforms that collect recurring information about their customers, who either do not have other alternatives or face substantial switching costs. Consider Facebook in the online social networking industry, Amazon prime in the retail sector, or Google Adsense in online advertising. They are all close to a monopoly in their respective industries. However, in industries where dominance is not yet established, competition for consumers may potentially alter our analysis considerably. For instance, consumers with higher valuations may be better off revealing their identities to induce head-to-head competition and get better prices. We leave the study of consumer profiling under competition for future research.

\section{References}

Acquisti, A., C. Taylor, L. Wagman. 2016. The Economics of Privacy. Journal of Economic Literature 54(2) $442-492$.

Acquisti, A., H. Varian. 2005. Conditioning Prices on Purchase History. Marketing Science 24(3) 367-381.

Belleflamme, P., W. Vergote. 2016. Monopoly Price Discrimination and Privacy: The Hidden Cost of Hiding. Economics Letters 149 141-144.

Casadesus-Masanell, R., A. Hervas-Drane. 2015. Competing with Privacy. Management Science 61(1) $229-246$.

Chen, Y., G. Iyer. 2002. Consumer Addressability and Customized Pricing. Marketing Science 21(2) 197208. 
Chen, Y., B. Jiang. 2019. Effects of Monitoring Technology on the Insurance Market. Production and Operations Management 28(8) 1957-1971.

Chen, Y., C. Narasimhan, Z. J. Zhang. 2001. Individual Marketing with Imperfect Targetability. Marketing Science 20(1) 23-41.

Chen, Y., Z. J. Zhang. 2009. Dynamic Targeted Pricing with Strategic Consumers. International Journal of Industrial Organization 27(1) 43-50.

Conger, K., R. Fausset, S.F. Kovaleski. 2019. San Francisco Bans Facial Recognition Technology. The New York Times, May $14^{\text {th }}$.

Conitzer, V., C. Taylor, L. Wagman. 2012. Hide and Seek: Costly Consumer Privacy in a Market with Repeat Purchases. Marketing Science 31(2) 277-292.

Esteves, R. 2009. A Survey on the Economics of Behaviour-Based Price Discrimination. NIPE Working Paper.

Federal Trade Commission. 2014. Data Brokers: A Call for Transparency and Accountability. FTC Report, Washington, D.C.

Fudenberg, D., J. Tirole. 2000. Customer Poaching and Brand Switching. RAND Journal of Economics 31(4) $634-657$.

Fudenberg, D., J. Villas-Boas. 2007. Behavior-Based Price Discrimination and Customer Recognition. T. Hendershott, ed., Handbook of Economics and Information Systems. Emerald.

Johnson, J. P., D. P. Myatt. 2006. On the Simple Economics of Advertising, Marketing, and Product Design. American Economic Review 96(3) 756-784.

Koh, B., S. Raghunathan, B. Nault. 2017. Is Voluntary Profiling Welfare Enhancing? MIS Quarterly 41(1) 23-41.

Lewis, L. 2019. Japan's Cash Addiction Will not be Easily Broken. The Financial Times, January $9^{\text {th }}$.

Montes, R., W. Sand-Zantman, T. Valletti. 2019. The Value of Personal Information in Online Markets with Endogenous Privacy. Management Science 65(3) 1342-1362.

Norvig, P. 2011. Internet-Scale Data Analysis. The annual ACM SIGKDD conference.

Pazgal, A., D. Soberman. 2008. Behavior-Based Discrimination: Is It a Winning Play, and If So, When? Marketing Science 27(6) 977-994. 
Shin, J., K. Sudhir. 2010. A Customer Management Dilemma: When is It Profitable to Reward One's Own Customers? Marketing Science 29(4) 671-689.

Shy, O., R. Stenbacka. 2016. Customer Privacy and Competition. Journal of Economics 6 Management Strategy 25(3) 539-562.

Singer, N. 2015. Sharing Data, but Not Happily. The New York Times, June $4^{\text {th }}$.

Singh, S. 2017. Competition in Corruptible Markets. Marketing Science 36(3) 361-381.

Taylor, C. 2004. Consumer privacy and the market for customer information. RAND Journal of Economics 35(4) 631-650.

Taylor, C., L. Wagman. 2014. Consumer Privacy in Oligopolistic Markets: Winners, Losers, and Welfare. International Journal of Industrial Organization 34(1) 80-84.

Villas-Boas, J. 1999. Dynamic Competition with Customer Recognition. RAND Journal of Economics 30(4) 604-631.

Villas-Boas, J. 2004. Price Cycles in Markets with Customer Recognition. RAND Journal of Economics 35(3) 486-501.

Wooldridge, J. 2010. Econometric Analysis of Cross Section and Panel Data. MIT Press.

Yang, Y. 2018. China's Data Privacy Outcry Fuels Case for Tighter Rules. The Financial Times, October $2^{\text {nd }}$.

Zhang, J. 2011. The Perils of Behavior-Based Personalization. Marketing Science 30(1) 170-186.

\section{APPENDIX: Proofs}

Proof of Lemma 1. It is straightforward to show the monotonicity of $v_{o}$ and $K_{o}$ in $\lambda$ and $c$, so we omit the proof here. Consider a separating equilibrium where there exists a cutoff $\tilde{v}$ such that a type- $v$ consumer in the old market would conceal if $v>\tilde{v}$, and she would otherwise reveal her identity when $v<\tilde{v}$. Denote the price that the firm offers to the anonymous market, i.e., those who conceal in the old market and the new market combined, as $p$. The expected revenue from charging $p$ to the anonymous market is given by

$$
\pi_{a}(p)=\lambda(1-\max \{p, \tilde{v}\}) p+(1-\lambda)(1-p) p .
$$

It is easy to verify that $p^{*}(\tilde{v})=1 / 2$ if $\tilde{v}<p$, and $p^{*}(\tilde{v})=\frac{1}{2}+\frac{\lambda(1-\tilde{v})}{2(1-\lambda)}$ when $\tilde{v} \geq p$. Consequently, when a type- $v$ consumer in the old market decides whether or not to conceal her identity, her expected utility of revealing is given by 


$$
u_{o}^{r}(v)=\max \{v-E[p(s) \mid v], 0\},
$$

where $p(s)$ is the price the firm charges upon receiving a signal $s$. Similarly, her expected utility of concealing is

$$
u_{o}^{c}(v)=\max \left\{v-p^{*}(\tilde{v}), 0\right\}-c .
$$

Given the cutoff $\tilde{v}$, we would require that $u_{o}^{c}(v)>u_{o}^{r}(v)$, for any $v>\tilde{v}$, and $u_{o}^{c}(v) \leq u_{o}^{r}(v)$, for any $v<\tilde{v}$.

First, we prove by contradiction that the price for the anonymous market $p$ must be less than the cutoff $\tilde{v}$ at equilibrium. Suppose that $p \geq \tilde{v}$. Consider a customer of type $\tilde{v}+\epsilon$, for any small $\epsilon>0$. Because $p \geq \tilde{v}$, we have $u_{o}^{c}(\tilde{v}+\epsilon)=\max \{\tilde{v}+\epsilon-p, 0\}-c<0$ due to the continuity of the utility function. Given that her expected utility from revealing is guaranteed to be non-negative, she is better-off revealing her identity, contradicting to the assumption that any customer with $v>\tilde{v}$ would choose to conceal. Thus, a necessary condition for the equilibrium is that $p \leq \tilde{v}$. Under this condition, we have $p^{*}(\tilde{v})=\frac{1}{2}+\frac{\lambda(1-\tilde{v})}{2(1-\lambda)}$, which implies that $\tilde{v} \geq \frac{1}{2-\lambda}$.

Next, we differentiate between the two cases, depending on how the firm's investment fares against $\tau(\tilde{v})$. The first case is when $K \geq \tau(\tilde{v})$. That is, the firm's investment is sufficiently large such that he can perfectly infer a customer's valuation if she chooses to reveal her identity. In this case, $E[p(s) \mid v]$ is reduced to $v$. Thus, the conditions that $u_{o}^{c}(v)>u_{o}^{r}(v)$, for any $v>\tilde{v}$, and $u_{o}^{c}(v) \leq u_{o}^{r}(v)$, for any $v<\tilde{v}$ can be written as

$$
\begin{aligned}
v-p^{*}(\tilde{v})-c>0, & \text { for any } v>\tilde{v}, \\
\max \left\{v-p^{*}(\tilde{v}), 0\right\}-c \leq 0, & \text { for any } v \leq \tilde{v} .
\end{aligned}
$$

The preceding inequalities can be reduced to $\tilde{v}-p^{*}(\tilde{v})-c=0$. Solving the equation, we have $\tilde{v}=v_{o} \equiv \frac{2 c(1-\lambda)+1}{2-\lambda} \geq \frac{1}{2-\lambda}$. Conversely, consider the following strategies and beliefs: the firm makes an investment $K \geq K_{o}$. Any customer in the old market with a valuation $v>v_{o}$ conceals, and the firm (correctly) infers their types, and charges $p^{*}(\tilde{v})=\frac{1}{2}+\frac{\lambda\left(1-v_{o}\right)}{2(1-\lambda)}$ to the anonymous market. Any customer in the old market with valuation $v \leq v_{o}$ reveals, and the firm receives a signal from each of those customers, and charges an individual price equal to the signal. Clearly, these strategies and beliefs form a PBE, so inequalities (2) and (3) are both sufficient as well as necessary for the existence of a separating equilibrium when the firm can profile those customers who choose to reveal perfectly.

Next consider the case when $\tau(1) \leq K<\tau(\tilde{v})$. In this case, as signals become uninformative, the firm's belief of valuation of a customer who chooses to reveal her identity follows a uniform 
distribution with support $[0, \tilde{v}]$. The optimal price the firm charges to this segment is given by $\arg \max _{p} p \int_{p}^{\tilde{v}} \frac{1}{\tilde{v}} d v=\frac{\tilde{v}}{2}$. The conditions that $u_{o}^{c}(v)>u_{o}^{r}(v)$, for any $v>\tilde{v}$, and $u_{o}^{c}(v) \leq u_{o}^{r}(v)$, for any $v<\tilde{v}$ can be written as

$$
\begin{array}{r}
v-p^{*}(\tilde{v})-c>\max \left\{v-\frac{\tilde{v}}{2}, 0\right\}, \quad \text { for any } v>\tilde{v}, \\
\max \left\{v-p^{*}(\tilde{v}), 0\right\}-c \leq \max \left\{v-\frac{\tilde{v}}{2}, 0\right\}, \quad \text { for any } v \leq \tilde{v} .
\end{array}
$$

However, the first inequality is always violated for any $\tilde{v}<\tau^{-1}(K)$. That is, a customer in the old market would always choose to reveal her identity up until the point that her decision of revealing leads to perfect profiling from the firm. As such, we have $\tilde{v}=\tau^{-1}(K)$. In this case, the conditions that $u_{o}^{c}(v)>u_{o}^{r}(v)$, for any $v>\tilde{v}$, and $u_{o}^{c}(v) \leq u_{o}^{r}(v)$, for any $v<\tilde{v}$ can be written as

$$
\begin{aligned}
v-p^{*}(\tilde{v})-c & >0, \quad \text { for any } v \geq \tilde{v}, \\
\max \left\{v-p^{*}(\tilde{v}), 0\right\}-c & \leq \max \left\{v-\frac{\tilde{v}}{2}, 0\right\}, \quad \text { for any } v<\tilde{v} .
\end{aligned}
$$

The preceding conditions can be reduced to $\tilde{v}>v_{o}$, which is satisfied if and only if $K<K_{o}$. Conversely, consider the following strategies and beliefs: the firm makes an investment $K<K_{o}$. Any customer in the old market with valuation $v \geq \tau^{-1}(K)$ conceals, and the firm (correctly) infers their types, and charges $p^{*}\left(v_{K}\right)=\frac{1}{2}+\frac{\lambda\left[1-\tau^{-1}(K)\right]}{2(1-\lambda)}$ to the anonymous market. Any customer in the old market with valuation $v<\tau^{-1}(K)$ reveals, and the firm (correctly) infers their types and charges $\tau^{-1}(K) / 2$ to those who reveal their identities. Clearly, these strategies and beliefs form a PBE, so inequalities (4) and (5) are both sufficient as well as necessary for the existence of a separating equilibrium when $\tau(1) \leq K<K_{o}$.

Lastly, there is a trivial case when $K<\tau(1)$. In this case, all consumers in the old market would choose to reveal their identities, however signals from those consumers are completely uninformative. Thus, the firm faces two market segments, namely, the new market and the old market, and consumer valuations from each segment are uniformly distributed on the unit interval. Consequently, the firm would offer the optimal monopoly price of $1 / 2$ to both the new market and the old market. We thus obtain the announced results.

Proof of Lemma 2. When the firm's investment level $K$ is greater than or equal to $K_{o}$, any consumer in the old market with a valuation greater than $v_{o}$ would choose to conceal her identity. The price the firm charges to the anonymous market is given by $p^{*}\left(v_{o}\right)=\frac{1-\lambda c}{2-\lambda}$, as characterized in Lemma 1. Consequently, the firm's profit with an investment of $K$ is given by

$$
\pi_{K}(\lambda, c)=\lambda\left(\int_{0}^{v_{o}} v d v+\int_{v_{o}}^{1} p^{*}\left(v_{o}\right) d v\right)+(1-\lambda) \int_{p^{*}\left(v_{o}\right)}^{1} p^{*}\left(v_{o}\right) d v-K
$$




$$
=\frac{1}{2(2-\lambda)}+\frac{\lambda c^{2}(1-\lambda)}{2-\lambda}-K
$$

When $K<K_{o}$, any consumer in the old market with a valuation greater than or equal to $v_{K}$ would choose to conceal her identity. The price the firm charges to those anonymous consumers is given by $p^{*}\left(v_{K}\right)=\frac{1}{2}+\frac{\lambda\left(1-v_{K}\right)}{2(1-\lambda)}$, as characterized in Lemma 1. Consequently, the firm's profit, for any $K \in\left[\tau(1), K_{o}\right)$, is

$$
\begin{aligned}
\pi_{K}(\lambda, c) & =\lambda\left(\int_{v_{K} / 2}^{v_{K}} \frac{v_{K}}{2} d v+\int_{v_{K}}^{1} p^{*}\left(v_{K}\right) d v\right)+(1-\lambda) \int_{p^{*}\left(v_{K}\right)}^{1} p^{*}\left(v_{K}\right) d v-K \\
& =\frac{\lambda\left(v_{K}-1\right)^{2}}{4(1-\lambda)}+\frac{1}{4}-K .
\end{aligned}
$$

In particular, contribution of revenue from those who choose to reveal their identities is $\lambda \int_{v_{K} / 2}^{v_{K}} \frac{v_{K}}{2} d v=\frac{\lambda v_{K}^{2}}{4}$, and contribution of revenue from the anonymous market is $\lambda \int_{v_{K}}^{1} p^{*}\left(v_{K}\right) d v+$ $(1-\lambda) \int_{p^{*}\left(v_{K}\right)}^{1} p^{*}\left(v_{K}\right) d v=\frac{\left(1-\lambda v_{K}\right)^{2}}{4(1-\lambda)}$.

When $K<\tau(1)$, the firm is not able to gain any information from profiling even if all consumers in the old market choose to reveal their identities. Thus, all consumers in the old market would be better-off revealing their identities, and the firm essentially faces two market segments, where consumers' valuations are uniformly distributed on the unit interval $[0,1]$ within each segment. Thus the firm's expected profit is given by $\pi_{K}(\lambda, c)=\pi_{0}(\lambda, c)-K=1 / 4-K, \forall K \in[0, \tau(1))$. We thus obtain the announced result.

Proof of Lemma 3. Consider the following two scenarios: (1) $K \geq K_{o}$. From Lemma 2, we know that the firm gains no more information advantage once its investment reaches the threshold $K_{o}$. As a result, the firm's optimal investment level is given by $K_{o}, \forall K \geq K_{o}$; (2) $K<K_{o}$. The firm's profit function is given by $\pi_{K}(\lambda, c)=\frac{\lambda\left(v_{K}-1\right)^{2}}{4(1-\lambda)}+\frac{1}{4}-\tau\left(v_{K}\right), \forall v_{K} \in\left(v_{o}, 1\right)$. By the assumption that $\tau(\cdot)$ is a concave function, $\pi_{K}(\lambda, c)$ is a convex function in $v_{K}$, and thus the maximum is realized at the either end of the support. When the firm's investment is sufficiently close to $K_{o}$, revenue from those anonymous consumers is the same as that by investing $K_{o}$. However, the firm earns a revenue of $v_{o}^{2} / 2$ from those consumers revealing their identities with an investment of $K_{o}$ due to perfect profiling, but only half of that revenue with an investment sufficiently close to $K_{o}$. Thus, the firm is better-off investing $K_{o}$ than any investment $K_{o}-\epsilon$, for any small $\epsilon>0$. On the other hand, when $v_{K}=1$, the firm's optimal profit is given by $\pi_{0}(\lambda, c)=1 / 4$, which is greater than the firm's optimal profit with an investment sufficiently close to $\tau(1)$, which is $1 / 4-\tau(1)$. Thus we obtain the announced result. 
Proof of Proposition 1. (i) Taking the derivative of $\pi_{K_{o}}(\lambda, c)$ with respect to $c$, we have

$$
\frac{\partial \pi_{K_{o}}(\lambda, c)}{\partial c}=\frac{2 \lambda c(1-\lambda)}{2-\lambda}-\frac{\partial K_{o}}{\partial c} \geq 0
$$

where the inequality is due to $\gamma \in[0,1]$ and Lemma 1 . Similarly, taking the derivative of the profit function with respect to $\gamma$, we have

$$
\frac{\partial \pi_{K_{o}}(\lambda, c)}{\partial \lambda}=\frac{1+\lambda^{2} c^{2}-4 \lambda c^{2}}{2(2-\lambda)^{2}}+\frac{c^{2}}{2}-\frac{\partial K_{o}}{\partial \lambda} \geq 0,
$$

where the inequality is due to the assumption $c \leq 1 / 2, \gamma \in[0,1]$, and Lemma 1 .

(ii) Taking derivative of $C S_{K_{o}}(\lambda, c)$ with respect to $\lambda$, we have

$$
\begin{aligned}
\frac{\partial C S_{K_{o}}(\lambda, c)}{\partial \lambda} & =\frac{c^{2}\left(-\frac{3}{2} \lambda^{3}+9 \lambda^{2}-12 \lambda+4\right)+c\left(\lambda^{3}-6 \lambda^{2}+7 \lambda-2\right)+(\lambda-1)}{(2-\lambda)^{3}} \\
& \leq \frac{c^{2}\left(-\frac{3}{2} \lambda^{3}+9 \lambda^{2}-12 \lambda+4\right)+c\left(\lambda^{3}-6 \lambda^{2}+7 \lambda-2\right)+2 c(\lambda-1)}{(2-\lambda)^{3}} \\
& =\frac{c^{2}\left(-\frac{3}{2} \lambda^{3}+9 \lambda^{2}-12 \lambda+4\right)+c\left(\lambda^{3}-6 \lambda^{2}+9 \lambda-4\right)}{(2-\lambda)^{3}} \\
& \leq \frac{c^{2}\left(-\frac{3}{2} \lambda^{3}+9 \lambda^{2}-12 \lambda+4\right)+2 c^{2}\left(\lambda^{3}-6 \lambda^{2}+9 \lambda-4\right)}{(2-\lambda)^{3}} \\
& =\frac{c^{2}\left(\frac{1}{2} \lambda^{3}-3 \lambda^{2}+6 \lambda-4\right)}{(2-\lambda)^{3}}<0 .
\end{aligned}
$$

The first inequality is due to $2 c \in[0,1]$ and $\lambda-1 \leq 0$. The second inequality is due to $2 c \in[0,1]$ and $g(\lambda) \equiv \lambda^{3}-6 \lambda^{2}+9 \lambda-4 \leq 0, \forall \lambda \in[0,1]\left(\right.$ as $d g(\lambda) / d \lambda=3(\lambda-2)^{2}-3 \geq 0, \forall \lambda \in[0,1]$ and $\left.g(1)=0\right)$. Similarly, it is easy to verify that $h(\lambda) \equiv \lambda^{3} / 2-3 \lambda^{2}+6 \lambda-4<0, \forall \lambda \in[0,1]$ since $d h(\lambda) / d \lambda=$ $3(\lambda-2)^{2} / 2 \geq 0, \forall \lambda \in[0,1]$ and $h(1)=-1 / 2<0$. Consequently, we conclude that $\partial C S_{K_{o}}(\lambda, c) / \partial \lambda<$ 0 .

The first and second-order derivatives of $C S_{K_{o}}(\lambda, c)$ with respect to $c$ are given by

$$
\begin{gathered}
\frac{\partial C S_{K_{o}}(\lambda, c)}{\partial c}=\lambda c-\lambda+\frac{\lambda(2 \lambda-3)(\lambda c-1)}{(\lambda-2)^{2}}, \\
\frac{\partial^{2} C S_{K_{o}}(\lambda, c)}{\partial c^{2}}=\frac{\lambda(3 \lambda-4)(\lambda-1)}{(\lambda-2)^{2}} \geq 0
\end{gathered}
$$

where the inequality is due to $\lambda \in[0,1]$. Consequently, $C S_{K_{o}}(\lambda, c)$ is convex in $c$.

(iii) The first and second-order derivatives of $S W_{K_{o}}(\lambda, c)$ with respect to $c$ are given by

$$
\begin{aligned}
\frac{\partial S W_{K_{o}}(\lambda, c)}{\partial c} & =\frac{\lambda\left[(8-5 \lambda)(1-\lambda) c-(\lambda-1)^{2}\right]}{(\lambda-2)^{2}}-\frac{\partial K_{o}}{\partial v_{o}} \cdot \frac{\partial v_{o}}{\partial c} \\
\frac{\partial^{2} S W_{K_{o}}(\lambda, c)}{\partial c^{2}} & =\frac{\lambda(1-\lambda)(8-5 \lambda)}{(\lambda-2)^{2}}-\frac{\partial^{2} K_{o}}{\partial v_{o}^{2}} \cdot\left(\frac{\partial v_{o}}{\partial c}\right)^{2}-\frac{\partial K_{o}}{\partial v_{o}} \cdot \frac{\partial^{2} v_{o}}{\partial c^{2}} .
\end{aligned}
$$


Given that $\lambda \in[0,1], \tau(\cdot)$ is concave, and $\partial^{2} v_{o} / \partial c^{2}=0, \partial^{2} S W_{K_{o}}(\lambda, c) / \partial c^{2}$ is guaranteed to be greater than or equal to 0 . Consequently, $S W_{K_{o}}(\lambda, c)$ is convex in $c$.

Similarly, the second-order derivative of $S W_{K_{o}}(\lambda, c)$ with respect to $\lambda$ is given by

$$
\begin{aligned}
\frac{\partial^{2} S W_{K_{o}}(\lambda, c)}{\partial \lambda^{2}} & =\frac{\left(16 c^{2}-10 c+1\right) \lambda+\left(-20 c^{2}+8 c+1\right)}{(\lambda-2)^{4}}-\frac{\partial^{2} K_{o}}{\partial v_{o}^{2}} \cdot\left(\frac{\partial v_{o}}{\partial \lambda}\right)^{2}-\frac{\partial K_{o}}{\partial v_{o}} \cdot \frac{2(1-2 c)}{(2-\lambda)^{3}} \\
& \geq \frac{\left(16 c^{2}-10 c+1\right) \lambda+\left(-20 c^{2}+8 c+1\right) \lambda}{(\lambda-2)^{4}}-\frac{\partial^{2} K_{o}}{\partial v_{o}^{2}} \cdot\left(\frac{\partial v_{o}}{\partial \lambda}\right)^{2}-\frac{\partial K_{o}}{\partial v_{o}} \cdot \frac{2(1-2 c)}{(2-\lambda)^{3}} \\
& =\frac{2 \lambda(1-2 c)(1+c)}{(\lambda-2)^{4}}-\frac{\partial^{2} K_{o}}{\partial v_{o}^{2}} \cdot\left(\frac{\partial v_{o}}{\partial \lambda}\right)^{2}-\frac{\partial K_{o}}{\partial v_{o}} \cdot \frac{2(1-2 c)}{(2-\lambda)^{3}} \geq 0
\end{aligned}
$$

The first inequality is due to $\lambda \in[0,1]$, and $-20 c^{2}+8 c+1=(1-2 c)(1+10 c) \geq 0$ because of $c \in[0,1 / 2]$. The second inequality is due to $c \in[0,1 / 2], \lambda \in[0,1]$, and $\tau(\cdot)$ is non-increasing and concave. We thus obtain the announced result.

Proof of Corollary 1. (i) It is easy to verify that $\partial C S_{K_{o}}(\lambda, c) / \partial \lambda<0$, and $C S_{K_{o}}(\lambda, c)$ is maximized at $\lambda=0$, where it attains a value of $1 / 8$. Recall that $C S_{0}(\lambda, c)=1 / 8$. We thus have $C S_{K_{o}}(\lambda, c) \leq C S_{0}(\lambda, c)$ for any $\lambda$ and $c$.

(ii) We seek to establish the inequality by showing that $S W_{K_{o}}(\lambda, c) \geq S W_{0}(\lambda, c)$ for any $\lambda \geq 1 / 4$ when $K_{o}=0$, i.e., the function $\tau(\cdot)$ is extremely small irrespective of $\lambda$ and $c$. Recall that we show in Proposition 1(iii) that $S W_{K_{o}}(\lambda, c)$ is a convex function in $c$. This result remains valid when $K_{o}=0$, and its minimum is realized at $\partial S W_{K_{o}}(\lambda, c) / \partial c=0$, i.e., $c^{*}(\lambda)=\frac{1-\lambda}{8-5 \lambda}$. Plugging $c^{*}(\lambda)$ into $S W_{K_{o}}(\lambda, c)$, we have $S W_{K_{o}}\left(\lambda, c^{*}(\lambda)\right)=\frac{\lambda^{2}-4 \lambda+6}{2(8-5 \lambda)}$. The second-order derivative of $S W_{K_{o}}\left(\lambda, c^{*}(\lambda)\right)$ is given by

$$
\frac{d^{2} S W_{K_{o}}\left(\lambda, c^{*}(\lambda)\right)}{d \lambda^{2}}=\frac{54}{(8-5 \lambda)^{3}} \geq 0
$$

where the inequality is due to $\lambda \in[0,1]$. Consequently, $S W_{K_{o}}\left(\lambda, c^{*}(\lambda)\right)$ is convex in $\lambda$. The two roots such that $S W_{K_{o}}\left(\lambda, c^{*}(\lambda)\right)=S W_{0}(\lambda, c)=3 / 8$ are given by $\lambda_{1}=0$ and $\lambda_{2}=1 / 4$. Consequently, $S W_{K_{o}}(\lambda, c) \geq 3 / 8$ for any $\lambda \geq 1 / 4$ when $K_{o}=0$, and we obtain the announced result.

Proof of Proposition 2. We use superscript $i=1,2$ to denote the two scenarios. Because $\tau_{1}(\gamma) \geq$ $\tau_{2}(\gamma), \forall \gamma \in[0,1], \pi_{K_{o}}^{1}(\lambda, c) \leq \pi_{K_{o}}^{2}(\lambda, c)$ for any $\lambda$ and $c$. Recall that $\pi_{0}^{1}(\lambda, c)=\pi_{0}^{2}(\lambda, c)=1 / 4$. Given different combinations of $\lambda$ and $c$, we have the following three cases.

(1) $\pi_{K_{o}}^{1}(\lambda, c) \leq \pi_{K_{o}}^{2}(\lambda, c) \leq 1 / 4$. Investing in consumer profiling is unprofitable for the firm under both $\tau_{1}$ and $\tau_{2}$. Consequently, the optimal decision for the firm is not to invest, which leads to $\pi^{1}(\lambda, c)=\pi^{2}(\lambda, c)=1 / 4, C S^{1}(\lambda, c)=C S^{2}(\lambda, c)=1 / 8$, and $S W^{1}(\lambda, c)=S W^{2}(\lambda, c)=3 / 8$. Recall that the firm's decision not to invest is always socially optimal. 
(2) $\pi_{K_{o}}^{1}(\lambda, c) \leq 1 / 4<\pi_{K_{o}}^{2}(\lambda, c)$. In this case, the firm invests in consumer profiling under $\tau_{2}$, but does not invest under $\tau_{1}$. As a result, $\pi^{2}(\lambda, c)=\pi_{K_{o}}^{2}(\lambda, c)>1 / 4=\pi_{0}^{1}(\lambda, c)=\pi^{1}(\lambda, c)$, and $C S^{2}(\lambda, c)=C S_{K_{o}}^{2}(\lambda, c) \leq C S_{0}^{2}(\lambda, c)=C S_{0}^{1}(\lambda, c)=C S^{1}(\lambda, c)$, where the inequality is given by Corollary 1. As shown in Lemma 4 , the firm's decision not to invest under $\tau_{1}$ is always socially optimal, however, the investment decision under $\tau_{2}$ may not be efficient if $\tau_{2}(\lambda, c)>\underline{K}_{\lambda, c}$.

(3) $1 / 4<\pi_{K_{o}}^{1}(\lambda, c) \leq \pi_{K_{o}}^{2}(\lambda, c)$. The firm invests in consumer profiling under both $\tau_{1}$ and $\tau_{2}$. Because $\tau_{1}(\gamma) \geq \tau_{2}(\gamma), \forall \gamma \in[0,1]$, we have $\pi^{1}(\lambda, c)=\pi_{K_{o}}^{1}(\lambda, c) \leq \pi_{K_{o}}^{2}(\lambda, c)=\pi^{2}(\lambda, c)$. Consumer surplus under the two scenarios are identical because it is independent of the scenarios should the firm decide to invest. Lastly, let us consider the efficiency of the firm's investment. If the investment is socially optimal under $\tau_{1}$, which indicates that $\tau_{1}(\lambda, c) \leq \underline{K}_{\lambda, c}$, then the investment decision is also socially optimal under $\tau_{2}$ because $\tau_{2}(\lambda, c) \leq \tau_{1}(\lambda, c) \leq \underline{K}_{\lambda, c}$. However the reverse is not true.

Summarizing the results from the three cases, we thus obtain the announced results. 


\section{Online Appendix \\ OA.1. Additional Results under General $\alpha_{K, \gamma}$}

In this section, we present additional results when $\alpha_{K, \gamma}$ follows a more general specification as follows:

$$
\alpha_{K, \gamma}=\frac{1}{1+\exp \left\{a\left(\gamma-b+K^{d}\right)\right\}}
$$

where $a \geq 0$ and $d \geq 0$.

\section{OA.1.1. Illustration of general $\alpha_{K, \gamma}$}

We first illustrate the shape of $\alpha_{K, \gamma}$ with different parameters in Figure OA.1. Figure OA.1(a) suggests that the parameter $a$ is associated with the curvature of $\alpha_{K, \gamma} . \alpha_{K, \gamma}$ rotates clockwise around the point $\gamma=b-K^{d}$ with a greater $a$. Parameters of $b$ and $d$ have similar effects, as illustrated in Figure OA.1(b) and (c), where changing the parameters shifts the curve either leftwards or rightwards. Based upon Definition 1, the curve corresponds to a scenario with higher data requirement if it shifts rightwards. Consequently, for two $b_{1}$ and $b_{2}, b_{1}$ indicates a scenario with higher data requirement than that of $b_{2}$ if $b_{1}>b_{2}$ and all the other parameters are the same. The effect of $d$ depends on the value of $K$. When $K<1$, a greater $d$ corresponds to a scenario with higher data requirement, whereas a smaller $d$ indicates higher data requirement when $K>1$.

Figure OA.1 Illustration of $\alpha_{K, \gamma}$ with Various $a, b$ and $d$.

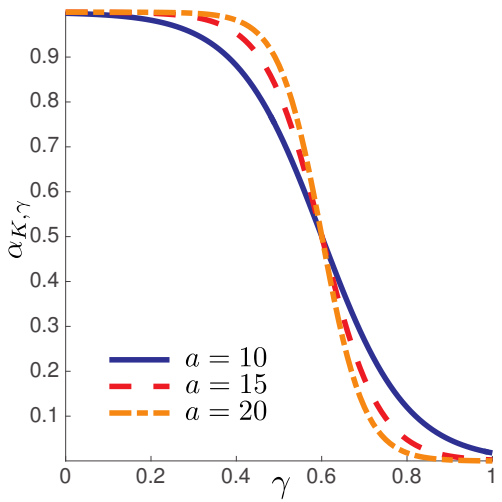

(a) Various $a$

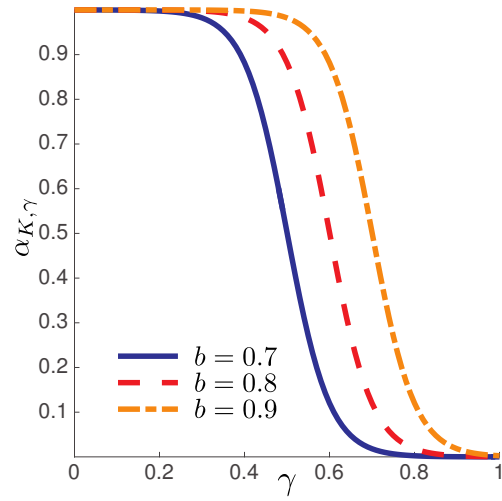

(b) Various $b$

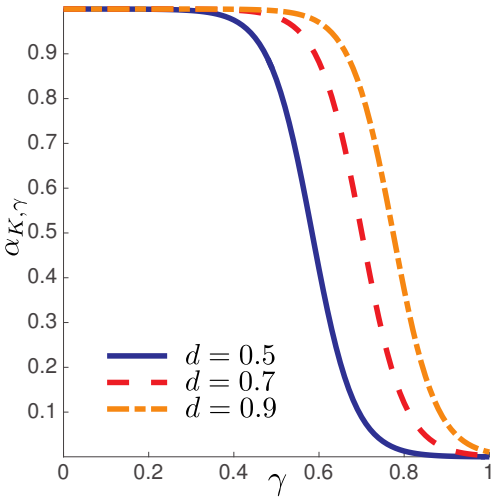

(c) Various $d$

Note. The parameters are specified as follows. $K=0.1, a=15, b=0.8$ and $d=0.7$ in the figures when the corresponding parameter is fixed. 


\section{OA.1.2. Equilibrium Analysis under General $\alpha_{K, \gamma}$}

Under the general $\alpha_{K, \gamma}$, the firm's belief of the valuation of a consumer upon receiving a signal $s$ is given by

$$
h(v \mid s)=\frac{1}{\min \left\{s+\alpha_{K, \gamma}, \tilde{v}\right\}-\max \left\{s-\alpha_{K, \gamma}, 0\right\}},
$$

for any $v \in\left[\max \left\{s-\alpha_{K, \gamma}, 0\right\}, \min \left\{s+\alpha_{K, \gamma}, \tilde{v}\right\}\right]$. The firm's posterior belief is uniformly distributed and centred around the signal $s$. The range of posterior valuations is narrower for the extreme signal values, i.e., when $s$ is close to 0 or $\tilde{v}$. This is consistent with the intuition that the firm is able to profile those consumers with extreme valuations more accurately.

Consequently, the firm's expected revenue from charging price $p$ to a consumer with signal $s$ is

$$
\pi_{s}(p)= \begin{cases}p, \quad \text { if } p<\max \left\{s-\alpha_{K, \gamma}, 0\right\}, \\ \frac{p\left(\min \left\{s+\alpha_{K, \gamma}, \tilde{v}\right\}-p\right)}{\min \left\{s+\alpha_{K, \gamma}, \tilde{v}\right\}-\max \left\{s-\alpha_{K, \gamma}, 0\right\}}, & \text { if } p \in\left[\max \left\{s-\alpha_{K, \gamma}, 0\right\}, \min \left\{s+\alpha_{K, \gamma}, \tilde{v}\right\}\right], \\ 0, & \text { if } p>\min \left\{s+\alpha_{K, \gamma}, \tilde{v}\right\} .\end{cases}
$$

It is easy to verify that the optimal price $p(s)$ that maximizes the firm's expected revenue is given by

$$
p(s)=\max \left\{\max \left\{s-\alpha_{K, \gamma}, 0\right\}, \frac{\min \left\{s+\alpha_{K, \gamma}, \tilde{v}\right\}}{2}\right\} .
$$

The optimal expected revenue from a consumer with signal $s$ is $\pi_{s}^{*}=\pi_{s}(p(s))$. Similarly, the expected consumer surplus when the firm charges $p(s)$ to a consumer with signal $s$ is given by

$$
\begin{aligned}
C S_{s}(p(s)) & =\int_{p(s)}^{\min \left\{s+\alpha_{K, \gamma}, \tilde{v}\right\}}(v-p(s)) \cdot h(v \mid s) d v \\
& =\frac{\left(\min \left\{s+\alpha_{K, \gamma}, \tilde{v}\right\}-p(s)\right)^{2}}{2\left(\min \left\{s+\alpha_{K, \gamma}, \tilde{v}\right\}-\max \left\{s-\alpha_{K, \gamma}, 0\right\}\right)} .
\end{aligned}
$$

The price the firm charges those anonymous consumers remains the same as before, which is given by $p^{*}(\tilde{v})=\frac{1}{2}+\frac{\lambda(1-\tilde{v})}{2(1-\lambda)}$. Given $p(s)$ and $p^{*}(\tilde{v})$, we next derive the fraction of consumers in the old market who choose to reveal their identities at equilibrium. Consider the $\tilde{v}$-type consumer. As $s+\alpha_{K, \gamma} \geq \tilde{v}, p(s)$ for a $\tilde{v}$-type consumer is simplified to $p(s)=\max \left\{s-\alpha_{K, \gamma}, \frac{\tilde{v}}{2}\right\}, \forall s \in\left[\tilde{v}-\alpha_{K, \gamma}, \tilde{v}+\right.$ $\left.\alpha_{K, \gamma}\right]$. Consequently, the expected price for a $\tilde{v}$-type consumer if he chooses to reveal her identity is

$$
E[p(s) \mid \tilde{v}]=\int_{\tilde{v}-\alpha_{K, \gamma}}^{\tilde{v}+\alpha_{K, \gamma}} \frac{1}{2 \alpha_{K, \gamma}} p(s) d s= \begin{cases}\frac{\tilde{v}}{2}+\frac{\tilde{v}^{2}}{16 \alpha_{K, \gamma}} & \text { if } \tilde{v}<4 \alpha_{K, \gamma}, \\ \tilde{v}-\alpha_{K, \gamma} & \text { if } \tilde{v} \geq 4 \alpha_{K, \gamma} .\end{cases}
$$

Recall that $\tilde{v}$ is given by $E[p(s) \mid \tilde{v}]=p^{*}(\tilde{v})+c$. That is, when $\tilde{v} \leq 4 \alpha_{K, \gamma}, \tilde{v}$ is given by

$$
\frac{\tilde{v}}{2}+\frac{\tilde{v}^{2}}{16 \alpha_{K, \gamma}}=\frac{1}{2}+\frac{\lambda(1-\tilde{v})}{2(1-\lambda)}+c,
$$


and when $\tilde{v} \geq 4 \alpha_{K, \gamma}, \tilde{v}$ is given by

$$
\tilde{v}-\alpha_{K, \gamma}=\frac{1}{2}+\frac{\lambda(1-\tilde{v})}{2(1-\lambda)}+c
$$

Given $\tilde{v}$, the firm's profit from investing $K$ can thus be written as

$$
\begin{aligned}
\pi_{K}(\lambda, c)= & p^{*}(\tilde{v}) \cdot\left[\lambda(1-\tilde{v})+(1-\lambda)\left(1-p^{*}(\tilde{v})\right)\right]+\lambda \int_{-\alpha_{K, \gamma}}^{\tilde{v}+\alpha_{K, \gamma}} \int_{0}^{\tilde{v}} \frac{g(s \mid v)}{\tilde{v}} d v \cdot \pi_{s}(p(s)) d s-K \\
= & p^{*}(\tilde{v}) \cdot\left[\lambda(1-\tilde{v})+(1-\lambda)\left(1-p^{*}(\tilde{v})\right)\right] \\
& +\lambda \int_{-\alpha_{K, \gamma}}^{\tilde{v}+\alpha_{K, \gamma}} \frac{\left(\min \left\{s+\alpha_{K, \gamma}, \tilde{v}\right\}-\max \left\{s-\alpha_{K, \gamma}, 0\right\}\right)}{2 \alpha_{K, \gamma} \tilde{v}} \cdot \pi_{s}(p(s)) d s-K,
\end{aligned}
$$

where the first term and the second term on the right hand side of the equation indicate revenue from those anonymous consumers in both old and new markets, and revenue from those consumers who reveal their identities, respectively. The optimal investment level $K$ is the one that maximizes $\pi_{K}(\lambda, c)$. Similarly, the expected consumer surplus from investing $K$ is given by

$$
\begin{aligned}
C S_{K}(\lambda, c)= & \lambda \int_{\tilde{v}}^{1}\left(v-p^{*}(\tilde{v})-c\right) d v+(1-\lambda) \int_{p^{*}(\tilde{v})}^{1}\left(v-p^{*}(\tilde{v})\right) d v \\
& +\lambda \int_{-\alpha_{K, \gamma}}^{\tilde{v}+\alpha_{K, \gamma}} \int_{0}^{\tilde{v}} \frac{g(s \mid v)}{\tilde{v}} d v \cdot C S_{s}(p(s)) d s \\
= & \lambda(1-\tilde{v})\left(\frac{1+\tilde{v}}{2}-p^{*}(\tilde{v})-c\right)+(1-\lambda) \frac{\left(1-p^{*}(\tilde{v})\right)^{2}}{2} \\
& +\lambda \int_{-\alpha_{K, \gamma}}^{\tilde{v}+\alpha_{K, \gamma}} \frac{\left(\min \left\{s+\alpha_{K, \gamma}, \tilde{v}\right\}-p(s)\right)^{2}}{4 \alpha_{K, \gamma} \tilde{v}} d s
\end{aligned}
$$

and the total welfare $S W_{K}(\lambda, c)=\pi_{K}(\lambda, c)+C S_{K}(\lambda, c)$.

We can no longer characterize consumers' decisions on whether or not to conceal, and the firm's pricing decisions at equilibrium with closed-form analytical results. Thus, we resort to extensive numerical analysis below to illustrate how different forces would affect the firm's profit, consumer surplus and social welfare.

\section{OA.1.3. Numerical results}

Figure OA.2 shows how the optimal investment level $K$, the fraction of consumers choosing to reveal their identities $\gamma$, and the price for anonymous consumers $p^{*}(\tilde{v})$ are affected by parameters $c$ and $\lambda$. Interestingly, Figure OA.2(a) indicates that the firm's optimal investment level $K$ is generally not monotone in either $\lambda$ or $c$. However, when $\lambda$ is sufficiently large (i.e., $\lambda=0.7$ or $\lambda=0.9$ in the figure), the optimal investment level $K$ decreases in $c$. Similarly, when $c$ is sufficiently large $(c \geq 0.4$ in the figure), the optimal investment level also decreases in $\lambda$. This is consistent with our findings summarized in Lemma 1. Indeed, the monotonicity of $K$ under the special case is conditional on 
Figure OA.2 Impact of $c$ and $\lambda$ on Optimal Investment Level $K$, Fraction of Consumers choosing to Reveal Their Identities $\gamma$, and Price for Anonymous Consumers $p^{*}(\tilde{v})$.

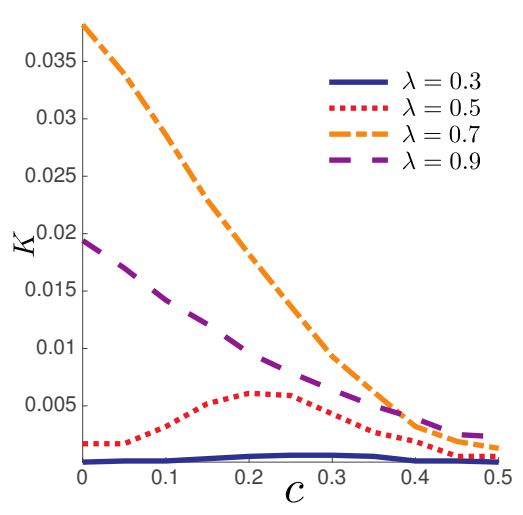

(a) $K$

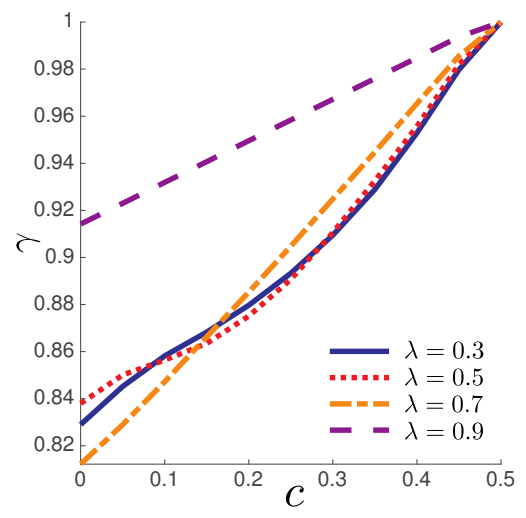

(b) $\gamma$

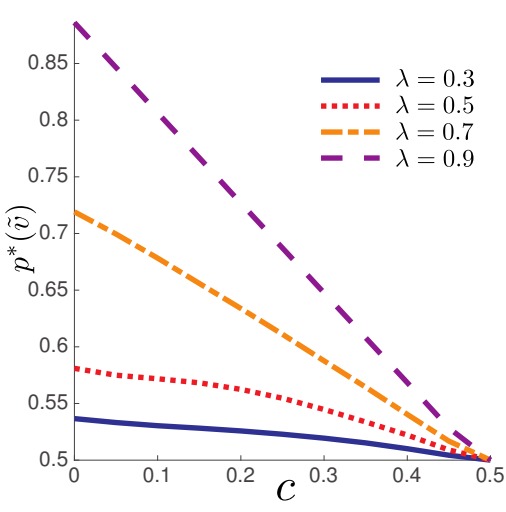

(c) $p^{*}(\tilde{v})$

Note. The parameters are specified as follows. $a=20, b=0.8$ and $d=0.7$.

the decision that the firm makes an investment, and the firm is shown to be more likely to invest with either a higher $c$ or a higher $\lambda$ by Proposition 1 .

When either $c$ or $\lambda$ is small, the monotonicity of $K$ no longer holds. Figure OA.2(a) suggests that $K$ first increases in $c$, and then decreases after a certain point for small $\lambda$. To understand this result, it is crucial to connect it with the endogenous decisions of consumers who can conceal their identities (see Figure OA.2(b)). When $c$ is small, it is almost costless for consumers to conceal, and most consumers would indeed choose to conceal should the firm decide to invest. Coupled with the fact that the old market is not large, the optimal investment from the firm would be small. On the other end of the spectrum, when $c$ is large, consumers have less flexibility in concealing their identities, and thus a small investment would be sufficient to extract all the benefits from profiling consumers. Small investments thus arise when it is either very easy or very difficult for consumers to protect their information. For intermediate values, the firm instead wants to invest more in the profiling technology, and thus the firm's investment is typically the highest for moderate $c$ when $\lambda$ is small.

The argument for the non-monotonicity of $\lambda$ when $c$ is small follows the same logic. When the old market is relatively small, the benefit of consumer profiling tends to be small, leading to a small investment level from the firm. However, when the old market is extremely large, a small investment is sufficient to guarantee that a good fraction of consumers would reveal their identities, allowing the firm to profile accurately. As such, the firm's optimal investment is the highest with a moderate-size old market $(\lambda=0.7)$. Figure OA.2(c) shows that the optimal price for anonymous 
consumers decreases in both $\lambda$ and $c$, which is a general property and a direct extension of the properties of $p^{*}\left(v_{o}\right)$ under the special case as given by Lemma 1 .

Figure OA.3 Impact of $c$ and $\lambda$ on Firm's Optimal Profit $\pi_{K}(\lambda, c)$, Consumer Surplus $C S_{K}(\lambda, c)$, and Social Welfare $S W_{K}(\lambda, c)$.

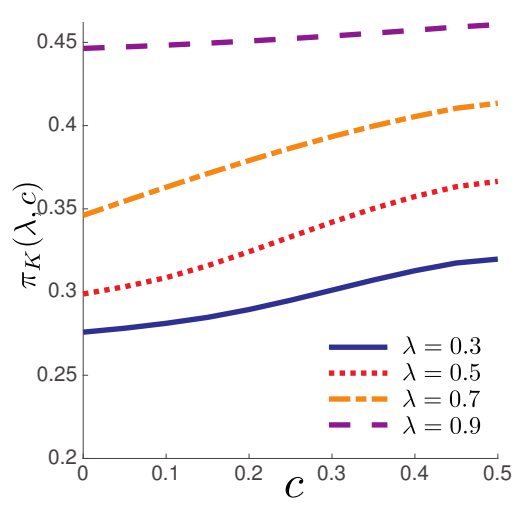

(a) $\pi_{K}(\lambda, c)$

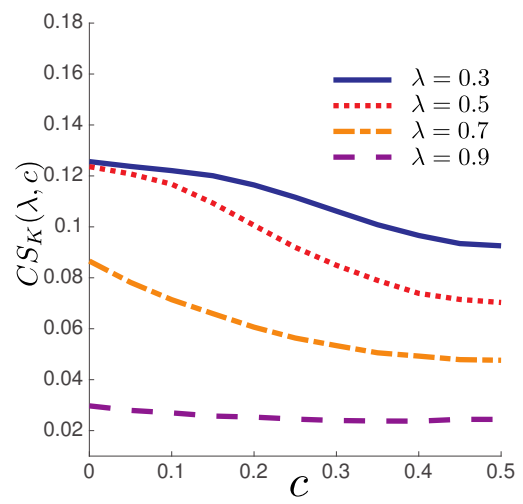

(b) $C S_{K}(\lambda, c)$

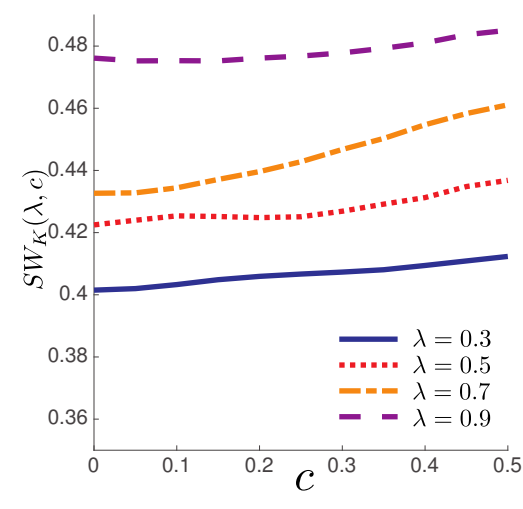

(c) $S W_{K}(\lambda, c)$

Note. The parameters are specified as follows. $a=20, b=0.8$ and $d=0.7$.

Figure OA.3 illustrates the impact of $c$ and $\lambda$ on the firm's optimal profit $\pi_{K}(\lambda, c)$, consumer surplus $C S_{K}(\lambda, c)$, and social welfare $S W_{K}(\lambda, c)$. Consistent with Proposition 1, the firm's optimal profit $\pi_{K}(\lambda, c)$ always increases in $c$ and $\lambda$. Again, the monotonicity result in Proposition 1 is conditional on the firm's decision to invest which is more likely to happen with either a higher $c$ or a higher $\lambda$. Figure OA.3(a) suggests that the monotonicity result holds generally, even for small $c$ and small $\lambda$.

Figure OA.3(b) indicates that consumer surplus decreases in $\lambda$, which is consistent with our result in Proposition 1(ii). With a larger old market, consumer profiling always becomes more attractive to the firm as it can price discriminate a larger fraction of the market, leading to a higher profit and lower consumer surplus. On the other hand, consumer surplus generally decreases in $c$. However, this result does not always hold. When $\lambda=0.9$, consumer surplus first decreases, and then increases in $c$ (this may not be obvious in Figure OA.3(b), simply due to scale). Indeed this convexity of consumer surplus in $c$ is already present in Proposition 1(ii). Under the special case when $\alpha_{K, \gamma}$ is a step function, social welfare is convex in both $\lambda$ and $c$ as shown in Proposition 1(iii). Its non-monotonicity in $c$ still holds under the general scenario shown in Figure OA.3(c), however the total welfare seems to increase in $\lambda$ most of the time with the chosen parameter values. Total welfare typically reaches its maximum when privacy costs are extremely large (i.e., $c=0.5)$. This is 
because, in such case, consumers will avoid the expenditure of concealing their identity to protect their privacy, which is a pure wasteful activity in our model. Without protection, everyone in the old market gets profiled and output expands, which is positive for total efficiency. However, the downside is that all the consumer surplus in the old market is then appropriated by the firm, and therefore the distribution of total welfare among the parties is tilted heavily in favor of the firm.

Figure OA.4 Socially Optimal Investment $K_{s}$ under Various Model Parameters.

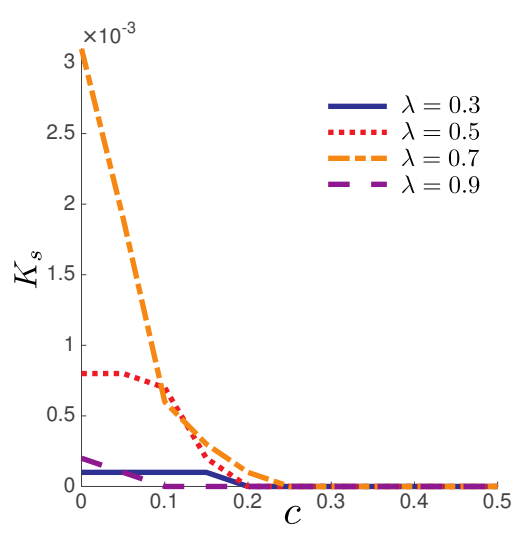

(a) $a=20, b=0.8, d=0.7$

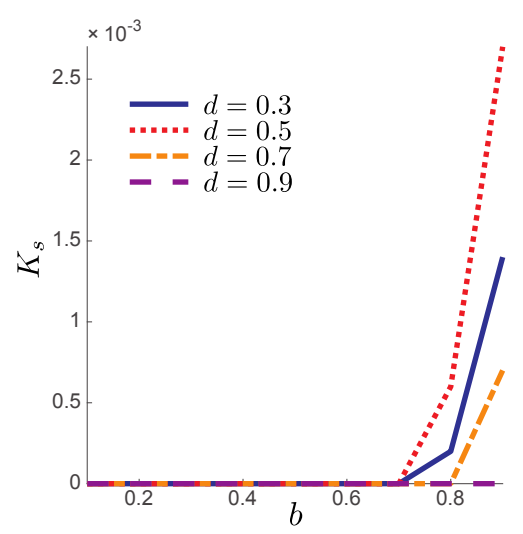

(b) $a=20, c=0.2, \lambda=0.5$

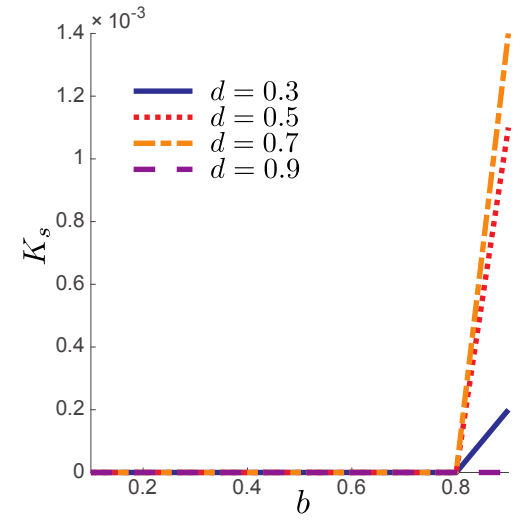

(c) $a=20, c=0.4, \lambda=0.9$

Note. Values of parameters which are fixed in the analysis are listed below the corresponding figures.

Lastly, we seek to find out under what conditions the firm's investment will be socially optimal. We expect the firm's investment to be generally suboptimal from a total welfare point of view, because the firm maximizes its profits without taking into account consumer surplus. The more interesting question we address here is the following: are efficiency concerns more acute in a "small data" or in a "big data" environment? In order to facilitate the comparison, we proceed as follows. First, we construct a benchmark where a central planner determines the investment with the goal of maximizing the total welfare. We denote the central planner's investment level as $K_{s}$. Once $K_{s}$ is decided, the model unfolds as discussed in our base model where the firm determines the price for anonymous consumers, as well as individual prices for each consumer who reveal her identity, and then consumers make their purchase decisions accordingly. Finally, we denote the difference in the investment levels from the firm and from the social planner as $\Delta K \equiv K-K_{s}$. This is a measure of relative (in)efficiency. A small $\Delta K$ implies that the private investment choice of the firm is aligned with social welfare. Conversely for large differences.

Figures OA.4 and OA.5 illustrate the optimal investment from the social planner $K_{s}$, and the 
difference between the social planner's investment and the firm's investment, respectively. In particular, Figure OA.5(a) illustrates the relative efficiency of the firm's investment with varying $c$ and $\lambda$. Recall that we show in Lemma 4 that the inefficiency interval increases in $\lambda$, and is concave in $c$ when $\alpha_{K, \gamma}$ is a step function. Figure OA.5(a) further corroborates this result under the general $\alpha_{K, \gamma}$. For a given $c$, the difference in the investments is generally smaller with smaller $\lambda$. The interval of $c$ where $\Delta K$ is greater than zero grows wider as $\lambda$ increases. On the other hand, for a given $\lambda$, the difference is minimized at $c=1 / 2$, and its maximum is realized with moderate $c$ when $\lambda=0.3$ or 0.5 . That is, $\Delta K$ is more likely to be zero under a wider range of values of $\lambda$ when $c$ is large compared with the situation when $c$ is moderate, which is consistent with the convexity property of the inefficiency interval as shown in Lemma 4.

Figure OA.5 Comparisons of Socially Optimal Investment $K_{s}$ and Optimal Monopoly Investment $K$ under Various Model Parameters.

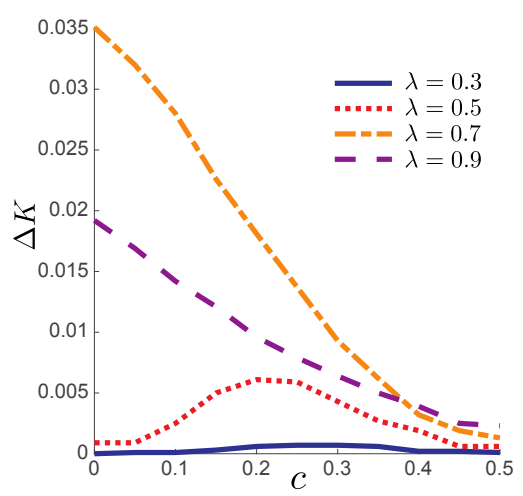

(a) $a=20, b=0.8, d=0.7$

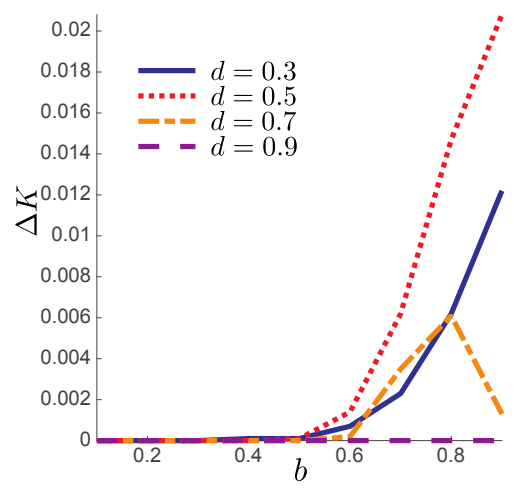

(b) $a=20, c=0.2, \lambda=0.5$

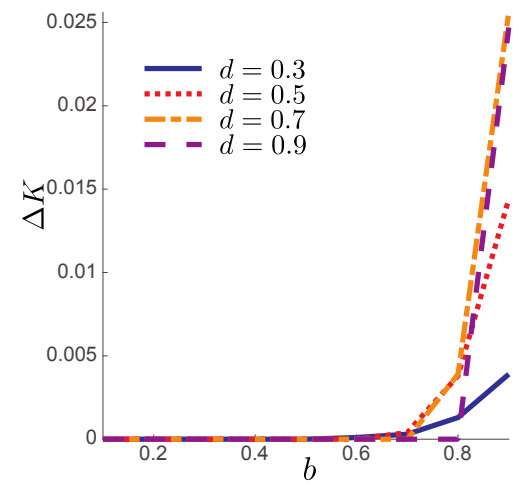

(c) $a=20, c=0.4, \lambda=0.9$

Note. Values of parameters which are fixed in the analysis are listed below the corresponding figures.

The impact of data requirement on the efficiency of the firm's investment is illustrated in Figure OA.5(b) and OA.5(c). In particular, we consider two scenarios, a scenario with moderate $\lambda$ and $c$ $(\lambda=0.5$ and $c=0.2)$, and a scenario with high $\lambda$ and $c(\lambda=0.9$ and $c=0.4)$. Under moderate $\lambda$ and $c$, the difference in the investments corresponding with higher data requirement, i.e., $d=0.7$ or 0.9 , is generally smaller than the difference under lower data requirement, i.e., $d=0.3$ or 0.5. However, this relationship is reversed in the scenario with high $\lambda$ and $c$. The numerical analysis provides evidence for Proposition 2(iii) under general $\alpha_{K, \gamma}$.

These welfare results can be read in two ways. When it is relatively easy (respectively, very costly) for consumers to protect their privacy, then higher data requirements (respectively, lower data 
requirements) cause less policy concerns. Alternatively, with lower data requirements (respectively, higher data requirements), the firm's investment decision is more likely aligned with the social welfare by making data protection very costly (respectively, relatively easy).

\section{OA.2. Proofs}

Proof of Lemma 4. From Corollary 1(i), we know that $C S_{K_{o}}(\lambda, c) \leq C S_{0}(\lambda, c)$ for any $\lambda$ and $c$, which is equivalent to $\pi_{K_{o}}(\lambda, c)-\pi_{0}(\lambda, c) \geq S W_{K_{o}}(\lambda, c)-S W_{0}(\lambda, c)$. It is easy to verify that $\pi_{K_{o}}(\lambda, c)-\pi_{0}(\lambda, c)=0$ if $K_{o}=\bar{K}_{\lambda, c}$, and $S W_{K_{o}}(\lambda, c)-S W_{0}(\lambda, c)=0$ if $K_{o}=\underline{K}_{\lambda, c} . \bar{K}_{\lambda, c} \geq \underline{K}_{\lambda, c}$ is guaranteed by the facts that $\pi_{\underline{K}_{\lambda, c}}(\lambda, c)-\pi_{0}(\lambda, c) \geq S W_{\underline{K}_{\lambda, c}}(\lambda, c)-S W_{0}(\lambda, c)=0=\pi_{\bar{K}_{\lambda, c}}(\lambda, c)-$ $\pi_{0}(\lambda, c)$, and $\pi_{K_{o}}(\lambda, c)$ is decreasing in $K_{o}$.

Consider the following three scenarios: (i) $K_{o}>\bar{K}_{\lambda, c}$, and thus $0>\pi_{K_{o}}(\lambda, c)-\pi_{0}(\lambda, c) \geq$ $S W_{K_{o}}(\lambda, c)-S W_{0}(\lambda, c)$. In this case, the firm gains a higher profit from not investing in consumer

profiling, and social welfare is also higher when the firm invests zero. (ii) $\underline{K}_{\lambda, c}<K_{o} \leq \bar{K}_{\lambda, c}$, and thus $\pi_{K_{o}}(\lambda, c)-\pi_{0}(\lambda, c) \geq 0>S W_{K_{o}}(\lambda, c)-S W_{0}(\lambda, c)$. The firm is better-off investing $K_{o}$ in consumer profiling, however social welfare is maximized when the firm invests zero. (iii) $K_{o} \leq \underline{K}_{\lambda, c}$, and thus $\pi_{K_{o}}(\lambda, c)-\pi_{0}(\lambda, c) \geq S W_{K_{o}}(\lambda, c)-S W_{0}(\lambda, c) \geq 0$. Both the firm's profit and social welfare are maximized when the firm invests $K_{o}$ in consumer profiling.

Lastly, we show the structural results of the interval $\bar{K}_{\lambda, c}-\underline{K}_{\lambda, c}$.

(1) The first-order derivative of $\bar{K}_{\lambda, c}-\underline{K}_{\lambda, c}$ with respect to $\lambda$ is given by

$$
\begin{aligned}
\frac{\partial\left(\bar{K}_{\lambda, c}-\underline{K}_{\lambda, c}\right)}{\partial \lambda} & =\frac{1}{2(\lambda-2)^{2}}\left[\left(-5 \lambda^{2}+10 \lambda-4\right) c^{2}+2(\lambda-1)^{2} c\right]+\frac{(\lambda c-1)^{2}(\lambda-1)}{(\lambda-2)^{3}} \\
& \geq \frac{1}{2(\lambda-2)^{2}}\left[\left(-5 \lambda^{2}+10 \lambda-4\right) c^{2}+2(\lambda-1)^{2} \cdot 2 c^{2}\right]+\frac{(\lambda c-1)^{2}(\lambda-1)}{(\lambda-2)^{3}} \\
& =\frac{\lambda c^{2}}{2(2-\lambda)}+\frac{(\lambda c-1)^{2}(\lambda-1)}{(\lambda-2)^{3}} \geq 0 .
\end{aligned}
$$

The first inequality is due to $2(\lambda-1)^{2} \geq 0$, and $c \in[0,1 / 2]$. The second inequality is due to $\lambda \in[0,1]$. Consequently, we conclude that $\left(\bar{K}_{\lambda, c}-\underline{K}_{\lambda, c}\right)$ is increasing in $\lambda$.

(2) The first and second-order derivatives of $\bar{K}_{\lambda, c}-\underline{K}_{\lambda, c}$ with respect to $c$ are given by

$$
\begin{aligned}
\frac{\partial\left(\bar{K}_{\lambda, c}-\underline{K}_{\lambda, c}\right)}{\partial c} & =\frac{\lambda(\lambda-1)[(4-3 \lambda) c+(\lambda-1)]}{(\lambda-2)^{2}}, \\
\frac{\partial^{2}\left(\bar{K}_{\lambda, c}-\underline{K}_{\lambda, c}\right)}{\partial c^{2}} & =\frac{\lambda(\lambda-1)(4-3 \lambda)}{(\lambda-2)^{2}} .
\end{aligned}
$$

Because $\lambda \in[0,1], \partial^{2}\left(\bar{K}_{\lambda, c}-\underline{K}_{\lambda, c}\right) / \partial c^{2} \leq 0$. Its maximum is realized at $c^{*}$, which is given by $\partial\left(\bar{K}_{\lambda, c}-\underline{K}_{\lambda, c}\right) / \partial c=0$, i.e., $c^{*}=(1-\lambda) /(4-3 \lambda)$. It is easy to verify that $c^{*}$ in decreasing in $\lambda$, and $c^{*} \in[0,1 / 4]$ as $\lambda \in[0,1]$. The minimum of $\bar{K}_{\lambda, c}-\underline{K}_{\lambda, c}$ is realized at either $c=0$ or $1 / 2$. When $c=0$, 
$\bar{K}_{\lambda, 0}-\underline{K}_{\lambda, 0}=\frac{3-2 \lambda}{2(2-\lambda)^{2}}-\frac{3}{8}$, and when $c=1 / 2, \bar{K}_{\lambda, 1 / 2}-\underline{K}_{\lambda, 1 / 2}=\frac{\lambda}{8}$. Taking difference of the two, we have

$$
\left(\bar{K}_{\lambda, 0}-\underline{K}_{\lambda, 0}\right)-\left(\bar{K}_{\lambda, 1 / 2}-\underline{K}_{\lambda, 1 / 2}\right)=\frac{\lambda^{2}(1-\lambda)}{8(2-\lambda)^{2}} \geq 0,
$$

where the inequality is due to $\lambda \in[0,1]$. We thus obtain the announced results.

Proof of Lemma 5. Consider a separating equilibrium where there exists a cutoff $\tilde{v}_{i}$ such that a type- $v$ consumer in the old market with concealing $\operatorname{cost} c_{i}$ would conceal if $v>\tilde{v}_{i}$, and she would otherwise reveal her identity when $v<\tilde{v}_{i}, i \in\{L, H\}$. Denote the price that the firm offers to the anonymous market, i.e., those who conceal in the old market and the new market combined, as $p$. The expected revenue from charging $p$ to the anonymous market is given by

$$
\pi_{a}(p)=\theta_{H} \lambda\left(1-\max \left\{p, \tilde{v}_{H}\right\}\right) p+\theta_{L} \lambda\left(1-\max \left\{p, \tilde{v}_{L}\right\}\right) p+(1-\lambda)(1-p) p .
$$

Similar that of the base model, it is easy to verify that necessary conditions for an equilibrium are that $\tilde{v}_{H} \geq p$ and $\tilde{v}_{L} \geq p$. As a result, the price that maximizes the firm's profit from the anonymous market is given by

$$
p^{*}\left(\tilde{v}_{H}, \tilde{v}_{L}\right)=\frac{1}{2}+\frac{\theta_{H} \lambda\left(1-\tilde{v}_{H}\right)+\theta_{L} \lambda\left(1-\tilde{v}_{L}\right)}{2(1-\lambda)} .
$$

When a type- $v$ consumer in the old market with concealing cost $c_{i}$ decides whether or not to conceal her identity, her expected utility of revealing is given by $u_{i}^{r}(v)=\max \{v-E[p(s) \mid v], 0\}$, where $p(s)$ is the price the firm charges upon receiving a signal $s$. Similarly, her expected utility of concealing is $u_{i}^{c}(v)=\max \left\{v-p^{*}\left(\tilde{v}_{H}, \tilde{v}_{L}\right), 0\right\}-c_{i}$. Given the cutoff $\tilde{v}_{i}$, we would require that $u_{i}^{c}(v)>u_{i}^{r}(v)$, for any $v>\tilde{v}_{i}$, and $u_{i}^{c}(v) \leq u_{i}^{r}(v)$, for any $v<\tilde{v}_{i}, i \in\{L, H\}$.

We differentiate between the two cases. The first case is when $K \geq \tau\left(\theta_{H} \tilde{v}_{H}+\theta_{L} \tilde{v}_{L}\right)$. That is, the firm's investment is sufficiently large such that he can perfectly infer a customer's valuation if she chooses to reveal her identity. In this case, $E[p(s) \mid v]$ is reduced to $v$. Thus, the conditions $u_{i}^{c}(v)>u_{i}^{r}(v)$, for any $v>\tilde{v}_{i}$, and $u_{i}^{c}(v) \leq u_{i}^{r}(v)$, for any $v<\tilde{v}_{i}, i \in\{L, H\}$, can be written as

$$
\begin{aligned}
v-p^{*}\left(\tilde{v}_{H}, \tilde{v}_{L}\right)-c_{i}>0, & \text { for any } v>\tilde{v}_{i}, \\
\max \left\{v-p^{*}\left(\tilde{v}_{H}, \tilde{v}_{L}\right), 0\right\}-c_{i} \leq 0, & \text { for any } v \leq \tilde{v}_{i} .
\end{aligned}
$$

The preceding inequalities can be reduced to $\tilde{v}_{i}-p^{*}\left(\tilde{v}_{H}, \tilde{v}_{L}\right)-c_{i}=0, i \in\{L, H\}$. Solving the equations, we have

$$
\tilde{v}_{i}=v_{o}^{i} \equiv \frac{1}{2}+c_{i}+\frac{\lambda}{2(2-\lambda)}\left(1-2 c_{H} \theta_{H}-2 c_{L} \theta_{L}\right), \quad i \in\{L, H\} .
$$

Denote $K_{o}^{\prime}=\tau\left(\theta_{H} v_{o}^{H}+\theta_{L} v_{o}^{L}\right)$. Conversely, consider the following strategies and beliefs: the firm makes an investment $K \geq K_{o}^{\prime}$. A type- $v$ customer in the old market with concealing $\operatorname{cost} c_{i}$ conceals 
if and only if $v>v_{o}^{i}, i \in\{L, H\}$, and the firm (correctly) infers their types, and charges $p^{*}\left(v_{o}^{H}, v_{o}^{L}\right)$ to the anonymous market. A type- $v$ customer in the old market with concealing $\operatorname{cost} c_{i}$ would reveal if $v \leq v_{o}^{i}, i \in\{L, H\}$, and the firm receives a signal from the customer, and charges an individual price equal to the signal. Clearly, these strategies and beliefs form a PBE, so inequalities (OA.2) and (OA.3) are both sufficient as well as necessary for the existence of a separating equilibrium when the firm can profile those customers who choose to reveal perfectly.

Next consider the case when $\tau(1)<K<\tau\left(\theta_{H} \tilde{v}_{H}+\theta_{L} \tilde{v}_{L}\right)$, i.e., $\tau^{-1}(K)>\theta_{H} \tilde{v}_{H}+\theta_{L} \tilde{v}_{L}$. In this case, valuations of customers who choose to reveal in the old market with concealing $\operatorname{cost} c_{i}$, follow a uniform distribution with support $\left[0, \tilde{v}_{i}\right], i \in\{L, H\}$. Due to $c_{H}>c_{L}$, we have $\tilde{v}_{H} \geq \tilde{v}_{L}$; Otherwise, a type- $\left(\tilde{v}_{L}-\epsilon\right)$ customer, for any small $\epsilon$, with concealing $\operatorname{cost} c_{L}$ shall receive a higher surplus by mimicking the decision of a type- $\left(\tilde{v}_{H}+\epsilon\right)$ customer with concealing $\operatorname{cost} c_{H}$, which contradicts to the definition of $\tilde{v}_{H}$ and $\tilde{v}_{L}$. In this case, signals are completely uninformative, and thus the firm changes one price, denoted as $p_{s}$, to all those who choose to reveal their identities. Consequently, the firm's profit from the revealing segment is given by

$$
\pi_{s}\left(p_{s}\right)=\theta_{H} \lambda\left(\tilde{v}_{H}-p_{s}\right) p_{s}+\theta_{L} \lambda\left(\tilde{v}_{L}-\min \left\{p_{s}, \tilde{v}_{L}\right\}\right) p_{s}
$$

When $p_{s}>\tilde{v}_{L}$, we have the optimal price $p_{s}^{*}=\tilde{v}_{H} / 2$, which holds under the condition that $\tilde{v}_{H}>2 \tilde{v}_{L}$. On the other hand, when $p_{s} \leq \tilde{v}_{L}$, we have $p_{s}^{*}=\frac{\theta_{H} \tilde{v}_{H}+\theta_{L} \tilde{v}_{L}}{2}$, which holds under the condition that $\theta_{H}\left(\tilde{v}_{H}-\tilde{v}_{L}\right) \leq \tilde{v}_{L}$

Consider first when $\tilde{v}_{H}>2 \tilde{v}_{L}$. In this case, the conditions $u_{i}^{c}(v)>u_{i}^{r}(v)$, for any $v>\tilde{v}_{i}$, and $u_{i}^{c}(v) \leq u_{i}^{r}(v)$, for any $v<\tilde{v}_{i}, i \in\{L, H\}$ can be written as

$$
\begin{aligned}
& v-p^{*}\left(\tilde{v}_{H}, \tilde{v}_{L}\right)-c_{H}>\max \left\{v-\frac{\tilde{v}_{H}}{2}, 0\right\}, \text { for any } v>\tilde{v}_{H}, \\
& \max \left\{v-p^{*}\left(\tilde{v}_{H}, \tilde{v}_{L}\right), 0\right\}-c_{H} \leq \max \left\{v-\frac{\tilde{v}_{H}}{2}, 0\right\}, \quad \text { for any } v \leq \tilde{v}_{H}, \\
& v-p^{*}\left(\tilde{v}_{H}, \tilde{v}_{L}\right)-c_{L}>0, \quad \text { for any } v>\tilde{v}_{L}, \\
& \max \left\{v-p^{*}\left(\tilde{v}_{H}, \tilde{v}_{L}\right), 0\right\}-c_{L} \leq 0, \quad \text { for any } v \leq \tilde{v}_{L} .
\end{aligned}
$$

Similar to that of the base model, it is easy to verify that the first inequality is always violated for any $\tilde{v}_{H}$. That is, when signals are uninformative, a customer with concealing $\operatorname{cost} c_{H}$ is always better off revealing her identity to mimic the behavior of low valuation customers. As such, customers with concealing $\operatorname{cost} c_{H}$ would always prefer to reveal her identity up until the point that her decision of revealing leads to perfect profiling from the firm. We thus have $\tau^{-1}(K)=\theta_{H} \tilde{v}_{H}+\theta_{L} \tilde{v}_{L}$. In this 
case, the conditions $u_{i}^{c}(v)>u_{i}^{r}(v)$, for any $v>\tilde{v}_{i}$, and $u_{i}^{c}(v) \leq u_{i}^{r}(v)$, for any $v<\tilde{v}_{i}, i \in\{L, H\}$, can be written as

$$
\begin{aligned}
& v-p^{*}\left(\tilde{v}_{H}, \tilde{v}_{L}\right)-c_{H}>0, \quad \text { for any } v \geq \tilde{v}_{H}, \\
& \max \left\{v-p^{*}\left(\tilde{v}_{H}, \tilde{v}_{L}\right), 0\right\}-c_{H} \leq \max \left\{v-\frac{\tilde{v}_{H}}{2}, 0\right\}, \quad \text { for any } v<\tilde{v}_{H}, \\
& v-p^{*}\left(\tilde{v}_{H}, \tilde{v}_{L}\right)-c_{L}>0, \quad \text { for any } v>\tilde{v}_{L}, \\
& \max \left\{v-p^{*}\left(\tilde{v}_{H}, \tilde{v}_{L}\right), 0\right\}-c_{L} \leq 0, \quad \text { for any } v \leq \tilde{v}_{L} .
\end{aligned}
$$

Solving the conditions $\tilde{v}_{L}-p^{*}\left(\tilde{v}_{H}, \tilde{v}_{L}\right)-c_{L}=0$ and $\tau^{-1}(K)=\theta_{H} \tilde{v}_{H}+\theta_{L} \tilde{v}_{L}$, we have $\tilde{v}_{L}=\frac{1}{2}+$ $\frac{\lambda\left[1-\tau^{-1}(K)\right]}{2(1-\lambda)}+c_{L}$. However, due to $\tau^{-1}(K) \in[0,1]$, we have $\tilde{v}_{H}>2 \tilde{v}_{L}>1$, which contradicts to $\tilde{v}_{H} \leq 1$. Thus, no equilibrium exists when $\tilde{v}_{H}>2 \tilde{v}_{L}$.

Next consider the case when $\theta_{H}\left(\tilde{v}_{H}-\tilde{v}_{L}\right) \leq \tilde{v}_{L}$. In this case, we have $p_{s}^{*}=\frac{\theta_{H} \tilde{v}_{H}+\theta_{L} \tilde{v}_{L}}{2}$. The conditions $u_{i}^{c}(v)>u_{i}^{r}(v)$, for any $v>\tilde{v}_{i}$, and $u_{i}^{c}(v) \leq u_{i}^{r}(v)$, for any $v<\tilde{v}_{i}, i \in\{L, H\}$ can be written as

$$
\begin{aligned}
v-p^{*}\left(\tilde{v}_{H}, \tilde{v}_{L}\right)-c_{i}>\max \left\{v-p_{s}^{*}, 0\right\}, & \text { for any } v>\tilde{v}_{i}, \\
\max \left\{v-p^{*}\left(\tilde{v}_{H}, \tilde{v}_{L}\right), 0\right\}-c_{i} \leq \max \left\{v-p_{s}^{*}, 0\right\}, & \text { for any } v \leq \tilde{v}_{i} .
\end{aligned}
$$

Again, it is easy to verify that the first inequality is always violated for any $\tilde{v}_{i}, i \in\{L, H\}$. That is, a customer in the old market would always prefer to reveal her identity up until the point that her decision of revealing leads to perfect profiling from the firm. As such, we have $\tau^{-1}(K)=$ $\theta_{H} \tilde{v}_{H}+\theta_{L} \tilde{v}_{L}$. In this case, the conditions $u_{i}^{c}(v)>u_{i}^{r}(v)$, for any $v>\tilde{v}_{i}$, and $u_{i}^{c}(v) \leq u_{i}^{r}(v)$, for any $v<\tilde{v}_{i}, i \in\{L, H\}$ can be written as

$$
\begin{gathered}
v-p^{*}\left(\tilde{v}_{H}, \tilde{v}_{L}\right)-c_{i}>0, \quad \text { for any } v \geq \tilde{v}_{i}, \\
\max \left\{v-p^{*}\left(\tilde{v}_{H}, \tilde{v}_{L}\right), 0\right\}-c_{i} \leq \max \left\{v-p_{s}^{*}, 0\right\}, \quad \text { for any } v<\tilde{v}_{i} .
\end{gathered}
$$

The preceding conditions can be reduced to $\tilde{v}_{H}>v_{o}^{H}, \tilde{v}_{L}>v_{o}^{L}$ and $\tau^{-1}(K)=\theta_{H} \tilde{v}_{H}+\theta_{L} \tilde{v}_{L}$. As we focus on symmetry equilibrium where $\tilde{v}_{H}=\tilde{v}_{L}$ when $c_{H}=c_{L}$, thus we have another condition that $\tilde{v}_{H}-p^{*}\left(\tilde{v}_{H}, \tilde{v}_{L}\right)-c_{H}=\tilde{v}_{L}-p^{*}\left(\tilde{v}_{H}, \tilde{v}_{L}\right)-c_{L}$.

Next we need to differentiate between two cases, depending on whether $K$ is greater than or less than $\tau\left(1-\theta_{L}\left(c_{H}-c_{L}\right)\right)$. When $\tau\left(1-\theta_{L}\left(c_{H}-c_{L}\right)\right) \leq K$, given that $\tau^{-1}(K)=\theta_{H} \tilde{v}_{H}+\theta_{L} \tilde{v}_{L}$, we have $\tilde{v}_{H}=v_{K}^{H} \equiv \tau^{-1}(K)+\theta_{L}\left(c_{H}-c_{L}\right)$ and $\tilde{v}_{L}=v_{K}^{L} \equiv \tau^{-1}(K)-\theta_{H}\left(c_{H}-c_{L}\right)$. The two inequalities $v_{K}^{H}>v_{o}^{H}$ and $v_{K}^{L}>v_{o}^{L}$ are satisfied if and only if $K<K_{o}^{\prime}$. The condition $\theta_{H}\left(\tilde{v}_{H}-\tilde{v}_{L}\right) \leq \tilde{v}_{L}$ is equivalent to 
$\tau^{-1}(K) \geq 2 \theta_{H}\left(c_{H}-c_{L}\right)$. Because $\tau^{-1}(K)=\theta_{H} v_{K}^{H}+\theta_{L} v_{K}^{L}>\theta_{H} v_{o}^{H}+\theta_{L} v_{o}^{L} \geq 1 / 2+\theta_{H} c_{H}+\theta_{L} c_{L} \geq$ $2 \theta_{H}\left(c_{H}-c_{L}\right)$, the condition is guaranteed to be satisfied when $\tau\left(1-\theta_{L}\left(c_{H}-c_{L}\right)\right) \leq K<K_{o}^{\prime}$.

When $\tau(1) \leq K<\tau\left(1-\theta_{L}\left(c_{H}-c_{L}\right)\right), v_{K}^{H}$ is equal to 1 . Thus, based on $\tau^{-1}(K)=\theta_{H} \tilde{v}_{H}+\theta_{L} \tilde{v}_{L}$, we have $\tau_{K}^{L}=\left(\tau^{-1}(K)-\theta_{H}\right) / \theta_{L}$. Following the same approach as before, it is easy to verify that the conditions $v_{K}^{H}>v_{o}^{H}, v_{K}^{L}>v_{o}^{L}$ and $\theta_{H}\left(v_{K}^{H}-v_{K}^{L}\right) \leq v_{K}^{L}$ are satisfied when $K<K_{o}^{\prime}$.

Conversely, consider the following strategies and beliefs: the firm makes an investment $\tau(1) \leq$ $K<K_{o}^{\prime}$. A type- $v$ customer in the old market with concealing cost $c_{i}$ conceals if and only if $v \geq v_{K}^{i}$, $i \in\{L, H\}$, and the firm (correctly) infers their types, and charges $p^{*}\left(v_{K}^{H}, v_{K}^{L}\right)$ to the anonymous market. A type- $v$ customer in the old market with concealing cost $c_{i}$ reveals if $v<v_{K}^{i}, i \in\{L, H\}$, and the firm (correctly) infers their types and charges $p_{s}^{*}$ to those who reveal their identities. Clearly, these strategies and beliefs form a PBE, so inequalities (OA.4) and (OA.5) are both sufficient as well as necessary for the existence of a separating equilibrium when signals are uninformative.

Lastly, there is a trivial case when $K<\tau(1)$. In this case, all consumers in the old market would choose to reveal their identities, however signals from those consumers are completely uninformative. Thus, the firm faces two market segments, namely, the new market and the old market, and consumer valuations from each segment are uniformly distributed on the unit interval. Consequently, the firm would offer the optimal monopoly price of $1 / 2$ to both the new market and the old market. We thus obtain the announced results.

Proof of Proposition 3. When the firm's investment level $K$ is greater than or equal to $K_{o}^{\prime}$, for any consumer in the old market with concealing cost $c_{i}$, she would conceal her identity if and only if $v>v_{o}^{i}, i \in\{L, H\}$. The firm receives a perfect signal for any consumer in the old market who chooses to reveal her identity, and charges an individual price equal to the signal. The price the firm charges to the anonymous market is given by $p^{*}\left(v_{o}^{H}, v_{o}^{L}\right)$, as characterized in Lemma 5(i). Consequently, the firm's profit is given by

$$
\begin{aligned}
\pi_{K}\left(\lambda, c_{H}, c_{L}\right)= & \theta_{H} \lambda\left(\int_{0}^{v_{o}^{H}} v d v+\int_{v_{o}^{H}}^{1} p^{*}\left(v_{o}^{H}, v_{o}^{L}\right) d v\right)+\theta_{L} \lambda\left(\int_{0}^{v_{o}^{L}} v d v+\int_{v_{o}^{L}}^{1} p^{*}\left(v_{o}^{H}, v_{o}^{L}\right) d v\right) \\
& +(1-\lambda) \int_{p^{*}\left(v_{o}^{H}, v_{o}^{L}\right)}^{1} p^{*}\left(v_{o}^{H}, v_{o}^{L}\right) d v-K \\
= & \frac{1+\lambda(2-\lambda)\left(c_{L}^{2} \theta_{L}+c_{H}^{2} \theta_{H}\right)-\lambda^{2}\left(c_{L} \theta_{L}+c_{H} \theta_{H}\right)^{2}}{2(2-\lambda)}-K .
\end{aligned}
$$

Notice that the profit function is concave in $\theta_{H}$, with the unique maximizer $\theta_{H}^{*}=\frac{(2-\lambda) c_{H}+(2-3 \lambda) c_{L}}{2 \lambda\left(c_{H}-c_{L}\right)}$. So when either $\lambda \leq 2 / 3$ or $\lambda>2 / 3$ and $c_{H} \leq \frac{2-\lambda}{3 \lambda-2} c_{L}$, we have $\theta_{H}^{*} \geq 1$, and thus the firm's profit always increases in $\theta_{H}$. However, when $\lambda>2 / 3$ and $c_{H}>\frac{2-\lambda}{3 \lambda-2} c_{L}$, which implies $\theta_{H}^{*}<1$, the firm's profit first increases but then decreases in $\theta_{H}$ once $\theta_{H}$ becomes greater than the critical point $\theta_{H}^{*}$. 
Second, consider the case when $K \in\left[\tau\left(1-\theta_{L}\left(c_{H}-c_{L}\right)\right), K_{o}^{\prime}\right)$. A consumer with concealing cost $c_{i}$ would conceal her identity if and only if $v>v_{o}^{i}, i \in\{L, H\}$. Signals are non-informative, and thus the firm would charge $p^{*}\left(v_{K}^{H}, v_{K}^{L}\right)$ and $p_{s}^{*}$ to the anonymous market and to those who reveal their identities, respectively. Consequently, the firm's profit is given by

$$
\begin{aligned}
\pi_{K}\left(\lambda, c_{H}, c_{L}\right)= & \theta_{H} \lambda\left(\int_{p_{s}^{*}}^{v_{K}^{H}} p_{s}^{*} d v+\int_{v_{K}^{H}}^{1} p^{*}\left(v_{K}^{H}, v_{K}^{L}\right) d v\right)+\theta_{L} \lambda\left(\int_{p_{s}^{*}}^{v_{K}^{L}} p_{s}^{*} d v+\int_{v_{K}^{L}}^{1} p^{*}\left(v_{K}^{H}, v_{K}^{L}\right) d v\right) \\
& +(1-\lambda) \int_{p^{*}\left(v_{K}^{H}, v_{K}^{L}\right)}^{1} p^{*}\left(v_{K}^{H}, v_{K}^{L}\right) d v-K \\
= & \frac{\lambda\left(\tau^{-1}(K)-1\right)^{2}}{4(1-\lambda)}+\frac{1}{4}-K .
\end{aligned}
$$

Similarly, when $K \in\left[\tau(1), \tau\left(1-\theta_{L}\left(c_{H}-c_{L}\right)\right)\right)$, the prices charged to the anonymous market and to those who reveal are characterized in Lemma 5(iii). Thus, we can derive the firm's expected profit as follows.

$$
\begin{aligned}
\pi_{K}\left(\lambda, c_{H}, c_{L}\right)= & \theta_{H} \lambda \int_{p_{s}^{*}}^{1} p_{s}^{*} d v+\theta_{L} \lambda\left(\int_{p_{s}^{*}}^{v_{K}^{L}} p_{s}^{*} d v+\int_{v_{K}^{L}}^{1} p^{*}\left(v_{K}^{H}, v_{K}^{L}\right) d v\right) \\
& +(1-\lambda) \int_{p^{*}\left(v_{K}^{H}, v_{K}^{L}\right)}^{1} p^{*}\left(v_{K}^{H}, v_{K}^{L}\right) d v-K \\
= & \frac{\lambda\left(\tau^{-1}(K)-1\right)^{2}}{4(1-\lambda)}+\frac{1}{4}-K .
\end{aligned}
$$

When $K<\tau(1)$, the situation would be exactly the same as the base model. The firm is not able to gain any information from profiling even if all consumers in the old market choose to reveal their identities. Thus, all consumers in the old market would be better-off revealing their identities, and the firm essentially faces two market segments, where consumers' valuations are uniformly distributed on the unit interval $[0,1]$ within each segment. Thus the firm's expected profit is given by $\pi_{K}(\lambda, c)=1 / 4-K, \forall K \in[0, \tau(1))$. We thus obtain the announced result.

Proof of Lemma 6. Consider two cases. The first case is when $K \geq \tau(\tilde{v})$. In this case, the firm receives a perfect signal from any customer who chooses to reveal her identity, and charges an individual price equal to the signal. The uniform price offered to the anonymous market is given in Lemma 1. Thus, the firm's expected profit, given any investment level $K$, is

$$
\begin{aligned}
\pi^{p}(K ; \tilde{v}) & =\lambda\left(\int_{0}^{\tilde{v}} v d v+\int_{\tilde{v}}^{1} p^{*}(\tilde{v}) d v\right)+(1-\lambda) \int_{p^{*}(\tilde{v})}^{1} p^{*}(\tilde{v}) d v-K \\
& =\frac{\lambda(2-\lambda) \tilde{v}^{2}-2 \lambda \tilde{v}+1}{4(1-\lambda)}-K .
\end{aligned}
$$

It is easy to verify that the firm's optimal investment level is given by $K(\tilde{v})=\tau(\tilde{v})$, which yields a profit of $\pi^{p}(\tau(\tilde{v}) ; \tilde{v}) \equiv \pi^{p *}(\tilde{v})$. 
Next consider the case when $K<\tau(\tilde{v})$. In this case, signals are uninformative, and thus the firm offers a uniform price of $\tilde{v} / 2$ to those who reveal their identities. The price for the anonymous market is also given in Lemma 1. Thus, the firm's expected profit is given by

$$
\begin{aligned}
\pi^{n}(K ; \tilde{v}) & =\lambda\left(\int_{\tilde{v} / 2}^{\tilde{v}} \frac{\tilde{v}}{2} d v+\int_{\tilde{v}}^{1} p^{*}(\tilde{v}) d v\right)+(1-\lambda) \int_{p^{*}(\tilde{v})}^{1} p^{*}(\tilde{v}) d v-K \\
& =\frac{\lambda(\tilde{v}-1)^{2}}{4(1-\lambda)}+\frac{1}{4}-K
\end{aligned}
$$

Given the cutoff $\tilde{v}$, the firm's optimal investment level is simply $K(\tilde{v})=0$, which yields a profit of $\pi^{n}(0 ; \tilde{v}) \equiv \pi^{n *}(\tilde{v})$. Taking the difference of $\pi^{p *}(\tilde{v})$ and $\pi^{n *}(\tilde{v})$, we have

$$
\pi^{p *}(\tilde{v})-\pi^{n *}(\tilde{v})=\frac{\lambda \tilde{v}^{2}}{4}-\tau(\tilde{v})
$$

which is strictly increasing in $\tilde{v}, \forall \tilde{v} \in[0,1]$. Coupling with the observation that $\pi^{p *}(0)-\pi^{n *}(0)<0$, we thus obtain the announced result.

Proof of Proposition 4. We can derive the equilibrium by studying the best response curves from consumers and the firm, as characterized in Lemma 1 and Lemma 6, respectively. An illustrative example of best response curves are shown in Figure OA.6.

\section{Figure OA.6 Best Response Curves}

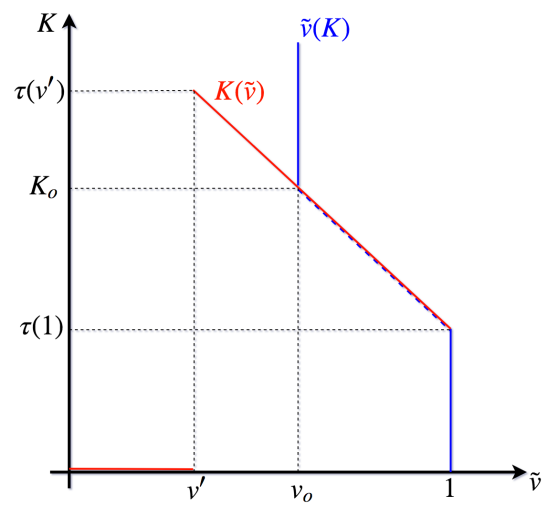

(a) $v^{\prime} \leq v_{o}$

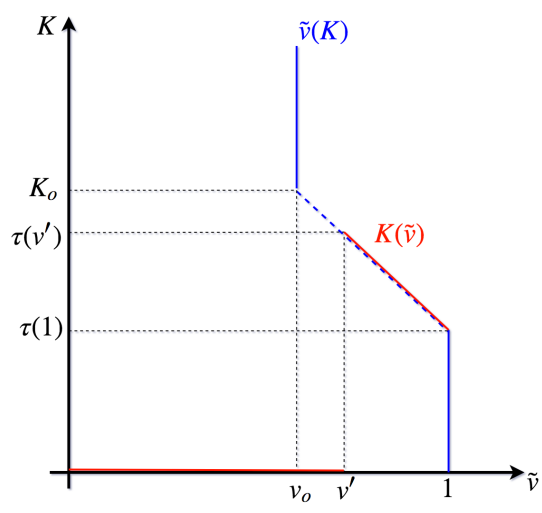

(b) $v^{\prime} \in\left(v_{o}, 1\right)$

Note. $K(\tilde{v})$ indicates the firm's optimal investment level anticipating that a type- $v$ consumer in the old market would conceal her identity if and only $v>\tilde{v} . \tilde{v}(K)$ indicates the optimal cutoff in the old market anticipating that the firm invests $K$ in profiling. For illustrative purpose, we assume that $\tau(\cdot)$ is linear in $\tilde{v}$ in the figure. However, our result in Proposition 4 holds for any non-increasing concave function $\tau(\cdot) . K_{o}$ and $v_{o}$ are given in Lemma 1 , and $v^{\prime}$ is given in Lemma 6. 
When $v^{\prime}$ is less than or equal to $v_{o}$, the firm prefers to invest in profiling even when a relatively small fraction of consumers in the old market choose to reveal their identities. In particular, anticipating that $v_{o}$ fraction of consumers choose to reveal, the firm would invest $K_{o}$ to profile them perfectly. On the other hand, anticipating that the firm's investment being greater than or equal to $K_{o}, v_{o}$ fraction of consumers would choose to reveal. Thus, the point $\left(v_{o}, K_{o}\right)$ in Figure OA.6(a) constitutes an equilibrium. This is also the unique equilibrium when $v^{\prime} \leq v_{o}$. This is because, for any $\tilde{v}>v_{o}$, the firm would find it profitable to perfectly profile those who reveal due to a lower required investment level. However, anticipating the firm's investment level being less than $K_{o}$, consumers' best response is characterized in Lemma 1(ii), where the fraction of consumers who choose to reveal is less than the level required to enable perfect profiling from the firm.

When $v^{\prime} \in\left(v_{o}, 1\right]$, the point $\left(v_{o}, K_{o}\right)$ no longer constitutes an equilibrium, as the firm would prefer not to invest at all when $v_{o}$ fraction of consumers in the old market reveal their identities. Following the same logic as that in the case when $v^{\prime} \leq v_{o}$, no equilibrium exists for any $K<K_{o}$. Thus, there exists no equilibrium when $v^{\prime} \in\left(v_{o}, 1\right]$.

Lastly, when $v^{\prime}>1$ or $v^{\prime}$ does not exist, the firm always prefers not to invest in profiling at all. On the other hand, anticipating the firm's investment being less than $\tau(1)$, all consumers in the old market would choose to reveal their identities. Thus, the point $(1,0)$ constitutes an equilibrium. It is easy to verify that this is also the unique equilibrium when $v^{\prime}>1$ or $v^{\prime}$ does not exist. We thus obtain the announced result. 